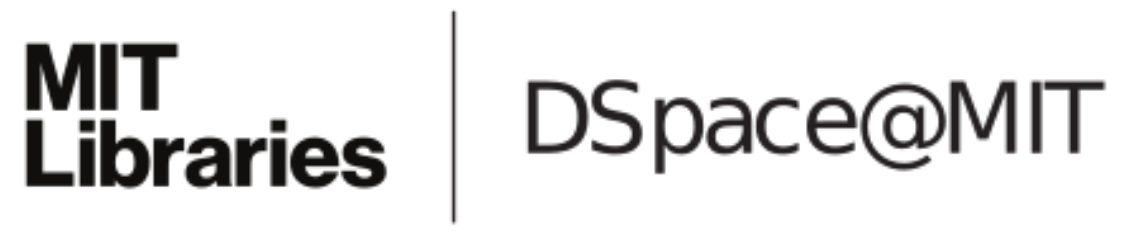

\author{
MIT Open Access Articles
}

Infeasibility Detection in the Alternating Direction

Method of Multipliers for Convex Optimization

The MIT Faculty has made this article openly available. Please share how this access benefits you. Your story matters.

As Published: https://doi.org/10.1007/s10957-019-01575-y

Publisher: Springer US

Persistent URL: https://hdl.handle.net/1721.1/131871

Version: Author's final manuscript: final author's manuscript post peer review, without publisher's formatting or copy editing

Terms of Use: Article is made available in accordance with the publisher's policy and may be subject to US copyright law. Please refer to the publisher's site for terms of use. 


\section{Infeasibility Detection in the Alternating Direction Method of Multipliers for Convex Optimization}

Cite this article as: Goran Banjac, Paul Goulart, Bartolomeo Stellato and Stephen Boyd, Infeasibility Detection in the Alternating Direction Method of Multipliers for Convex Optimization, Journal of Optimization Theory and Applications https://doi.org/10.1007/s10957019-01575-y

This Author Accepted Manuscript is a PDF file of an unedited peer-reviewed manuscript that has been accepted for publication but has not been copyedited or corrected. The official version of record that is published in the journal is kept up to date and so may therefore differ from this version.

Terms of use and reuse: academic research for non-commercial purposes, see here for full terms. https://www.springer.com/aam-terms-v1 


\title{
Infeasibility Detection in the Alternating Direction Method of Multipliers for Convex Optimization
}

\author{
Goran Banjac • Paul Goulart . \\ Bartolomeo Stellato · Stephen Boyd
}

Received: date / Accepted: date

\begin{abstract}
The alternating direction method of multipliers is a powerful operator splitting technique for solving structured optimization problems. For convex optimization problems, it is well-known that the algorithm generates iterates that converge to a solution, provided that it exists. If a solution does not exist, then the iterates diverge. Nevertheless, we show that they yield conclusive information regarding problem infeasibility for optimization problems with linear or quadratic objective functions and conic constraints, which includes quadratic, second-order cone, and semidefinite programs. In particular, we show that in the limit the iterates either satisfy a set of first-order optimality conditions or produce a certificate of either primal or dual infeasibility. Based on these results, we propose termination criteria for detecting primal and dual infeasibility.
\end{abstract}

Keywords Convex optimization · Infeasibility detection · Alternating direction method of multipliers · Conic programming

Mathematics Subject Classification (2000) 90C06 - 90C20 - 90C22 .

$90 \mathrm{C} 25 \cdot 68 \mathrm{Q} 25$

Communicated by Jalal M. Fadili.

Goran Banjac, Corresponding author

Automatic Control Laboratory, ETH Zurich, Zurich, Switzerland

E-mail: gbanjac@control.ee.ethz.ch

Paul Goulart

Department of Engineering Science, University of Oxford, Oxford, UK

E-mail: paul.goulart@eng.ox.ac.uk

Bartolomeo Stellato

Operations Research Center, Massachusetts Institute of Technology, Cambridge, MA, USA

E-mail: stellato@mit.edu

Stephen Boyd

Department of Electrical Engineering, Stanford University, Stanford, CA, USA

E-mail: boyd@stanford.edu 


\section{Introduction}

Operator splitting methods can be used to solve composite minimization problems where we minimize the sum of two convex, closed, and proper functions. These methods encompass algorithms such as the proximal gradient method (PGM), Douglas-Rachford splitting (DRS), and the alternating direction method of multipliers (ADMM) [1], and have been applied to problems ranging from feasibility and best approximation problems $[2,3]$ to quadratic and conic programs [4-6]. Due to their relatively low per-iteration computational cost and ability to exploit sparsity in the problem data [6], splitting methods are suitable for embedded [7-9] and large-scale optimization [10], and have increasingly been applied for solving problems arising in signal processing $[11,12]$, machine learning [13], and optimal control [14].

In order to solve a composite minimization problem, PGM requires differentiability of one of the two functions. If a fixed step-size is used in the algorithm, then one also requires a bound on the Lipschitz constant of the function's gradient [10]. On the other hand, ADMM and DRS, which turn out to be equivalent to each other, do not require any additional assumptions on the problem beyond convexity, making them more robust to the problem data.

The growing popularity of ADMM has triggered a strong interest in understanding its theoretical properties. Provided that a problem is solvable and satisfies certain constraint qualification (see [15, Cor. 26.3] for more details), both ADMM and DRS are known to converge to an optimal solution $[13,15]$. The use of ADMM for solving convex quadratic programs (QPs) was analyzed in [4] and was shown to admit an asymptotic linear convergence rate. The authors in [16] analyzed global linear convergence of ADMM for solving strongly convex QPs with inequality constraints that are linearly independent, and the authors in [17] extended these results to a wider class of optimization problems involving a strongly convex objective function. A particularly convenient framework for analyzing the asymptotic behavior of such method is by representing it as a fixed-point iteration of an averaged operator $[15,17,18]$.

The ability to detect infeasibility of an optimization problem is very important in many applications, e.g., in any embedded application or in mixedinteger optimization when branch-and-bound techniques are used [19]. It is well-known that for infeasible convex optimization problems some of the iterates of ADMM and DRS diverge [20]. However, terminating the algorithm, when the iterates become large, is unreliable in practice for several reasons. First, an upper bound on the allowed norm of the iterates should be sufficiently large so that the number of false detections of infeasibility is reduced. Second, divergence of the iterates is observed to be very slow in practice. Finally, such termination criterion is just an indication that a problem might be infeasible, and not a certificate of infeasibility.

Aside from [20], the asymptotic behavior of ADMM and DRS for infeasible problems has been studied only in some special cases. DRS for solving feasibility problems involving two convex sets, that do not necessarily intersect, was studied in [3,21-24]. The authors in [25] study the asymptotic behavior of 
ADMM for solving convex QPs when the problem is infeasible, but impose full rank assumptions on certain matrices derived from the problem data. The authors in [5] apply ADMM to the homogeneous self-dual embedding of a convex conic program, thereby producing a larger problem, which is always feasible and whose solutions can be used either to produce a primal-dual solution or a certificate of infeasibility for the original problem. A disadvantage of this approach in application to optimization problems with quadratic objective functions is that the problem needs to be transformed into an equivalent conic program, which is in general harder to solve than the original problem $[26,27]$.

In this paper we consider a class of convex optimization problems, that includes linear programs (LPs), QPs, second-order cone programs (SOCPs), and semidefinite programs (SDPs) as special cases. We use a particular version of ADMM, introduced in [28], that imposes no conditions on the problem data such as strong convexity of the objective function or full rank of the constraint matrix. We show that the method either generates iterates for which the violation of the optimality conditions goes to zero, or produces a certificate of primal or dual infeasibility. These results are directly applicable to infeasibility detection in ADMM for the considered class of problems.

We introduce some definitions and notation in Section 2, the problem of interest in Section 3, and present a particular ADMM algorithm for solving it in Section 4. Section 5 analyzes the asymptotic behavior of ADMM and shows that the algorithm can detect primal and dual infeasibility of the problem. Section 6 demonstrates these results on several small numerical examples. Finally, Section 7 concludes the paper.

\section{Notation}

All definitions introduced here are standard and can be found, e.g., in [15,29].

Let $\mathbb{N}$ denote the set of natural numbers, $\mathbb{R}$ the set of real numbers, $\mathbb{R}_{+}$ the set of non-negative real numbers, $\tilde{\mathbb{R}}:=\mathbb{R} \cup\{+\infty\}$ the extended real line, and $\mathbb{R}^{n}$ the $n$-dimensional real space equipped with inner product $\langle\cdot, \cdot\rangle$, induced norm $\|\cdot\|$, and identity operator Id: $x \mapsto x$. We denote by $\mathbb{R}^{m \times n}$ the set of real $m$-by- $n$ matrices and by $\mathbb{S}^{n}\left(\mathbb{S}_{+}^{n}\right)$ the set of real $n$-by- $n$ symmetric (positive semidefinite) matrices. Let vec: $\mathbb{S}^{n} \mapsto \mathbb{R}^{n^{2}}$ be the operator mapping a matrix to the stack of its columns, mat $=\mathrm{vec}^{-1}$ its inverse operator, and diag: $\mathbb{R}^{n} \mapsto \mathbb{S}^{n}$ the operator mapping a vector to a diagonal matrix. For a sequence $\left\{x^{k}\right\}_{k \in \mathbb{N}}$ we define $\delta x^{k+1}:=x^{k+1}-x^{k}$. The proximal operator of a convex, closed, and proper function $f: \mathbb{R}^{n} \mapsto \tilde{\mathbb{R}}$ is given by

$$
\operatorname{prox}_{f}(x):=\underset{y}{\operatorname{argmin}}\left\{f(y)+\frac{1}{2}\|y-x\|^{2}\right\} .
$$

For a nonempty, closed, and convex set $\mathcal{C} \subseteq \mathbb{R}^{n}$, we denote the indicator function of $\mathcal{C}$ by

$$
\mathcal{I}_{\mathcal{C}}(x):= \begin{cases}0, & x \in \mathcal{C} \\ +\infty, & \text { otherwise }\end{cases}
$$


the distance of $x \in \mathbb{R}^{n}$ to $\mathcal{C}$ by

$$
\operatorname{dist}_{\mathcal{C}}(x):=\min _{y \in \mathcal{C}}\|x-y\|,
$$

the projection of $x \in \mathbb{R}^{n}$ onto $\mathcal{C}$ by

$$
\Pi_{\mathcal{C}}(x):=\underset{y \in \mathcal{C}}{\operatorname{argmin}}\|x-y\|,
$$

the support function of $\mathcal{C}$ by

$$
S_{\mathcal{C}}(x):=\sup _{y \in \mathcal{C}}\langle x, y\rangle,
$$

the recession cone of $\mathcal{C}$ by

$$
\mathcal{C}^{\infty}:=\left\{y \in \mathbb{R}^{n}: x+\tau y \in \mathcal{C}, x \in \mathcal{C}, \tau \geq 0\right\},
$$

and the normal cone of $\mathcal{C}$ at $x \in \mathcal{C}$ by

$$
N_{\mathcal{C}}(x):=\left\{y \in \mathbb{R}^{n}: \sup _{x^{\prime} \in \mathcal{C}}\left\langle x^{\prime}-x, y\right\rangle \leq 0\right\} .
$$

Note that $\Pi_{\mathcal{C}}$ is the proximal operator of $\mathcal{I}_{\mathcal{C}}$. For a convex cone $\mathcal{K} \subseteq \mathbb{R}^{n}$, we denote its polar cone by

$$
\mathcal{K}^{\circ}:=\left\{y \in \mathbb{R}^{n}: \sup _{x \in \mathcal{K}}\langle x, y\rangle \leq 0\right\},
$$

and for any $b \in \mathbb{R}^{n}$ we denote a translated cone by $\mathcal{K}_{b}:=\mathcal{K}+\{b\}$.

Let $\mathcal{D}$ be a nonempty subset of $\mathbb{R}^{n}$. We denote the closure of $\mathcal{D}$ by $\operatorname{cl} \mathcal{D}$. For an operator $T: \mathcal{D} \mapsto \mathbb{R}^{n}$, we define its fixed-point set as

$$
\operatorname{Fix} T:=\{x \in \mathcal{D}: T x=x\},
$$

and denote its range by $\operatorname{ran}(T)$. We say that $T$ is nonexpansive if

$$
\|T x-T y\| \leq\|x-y\|, \quad \forall(x, y) \in \mathcal{D} \times \mathcal{D},
$$

and $T$ is $\alpha$-averaged with $\alpha \in] 0,1[$ if there exists a nonexpansive operator $R: \mathcal{D} \mapsto \mathbb{R}^{n}$ such that $T=(1-\alpha)$ Id $+\alpha R$.

\section{Problem Description}

Consider the following convex optimization problem:

$$
\min _{x}\left(\frac{1}{2} x^{T} P x+q^{T} x\right) \text { s.t. } A x \in \mathcal{C},
$$

with $P \in \mathbb{S}_{+}^{n}, q \in \mathbb{R}^{n}, A \in \mathbb{R}^{m \times n}$, and $\mathcal{C} \subseteq \mathbb{R}^{m}$ a nonempty, closed, and convex set. We make the following assumption on the set $\mathcal{C}$ :

Assumption 3.1 The set $\mathcal{C}$ is the Cartesian product of a convex and compact set $\mathcal{B} \subseteq \mathbb{R}^{m_{1}}$, and a translated closed and convex cone $\mathcal{K}_{b} \subseteq \mathbb{R}^{m_{2}}$, where $m_{1}$ and $m_{2}$ are non-negative integers and $m_{1}+m_{2}=m$, i.e., $\mathcal{C}=\mathcal{B} \times \mathcal{K}_{b}$.

Many convex problems of practical interest, including LPs, QPs, SOCPs, and SDPs, can be written in the form of problem (1) with $\mathcal{C}$ satisfying the conditions of Assumption 3.1. We are interested in finding either an optimal solution to problem (1) or a certificate of either primal or dual infeasibility. 


\subsection{Optimality Conditions}

We will find it convenient to rewrite problem (1) in an equivalent form by introducing a variable $z \in \mathbb{R}^{m}$ to obtain

$$
\min _{(x, z)}\left(\frac{1}{2} x^{T} P x+q^{T} x\right) \quad \text { s.t. } \quad A x=z \quad \text { and } \quad z \in \mathcal{C} .
$$

We can then write the optimality conditions for problem (2) as:

$$
\begin{aligned}
& A x-z=0 \\
& P x+q+A^{T} y=0 \\
& z \in \mathcal{C}, \quad y \in N_{\mathcal{C}}(z),
\end{aligned}
$$

where $y \in \mathbb{R}^{m}$ is a Lagrange multiplier associated with the constraint $A x=z$. If there exist $x \in \mathbb{R}^{n}, z \in \mathbb{R}^{m}$, and $y \in \mathbb{R}^{m}$ that satisfy conditions (3), then we say that $(x, z)$ is a primal and $y$ is a dual solution to problem (2). For completeness, we derive the optimality conditions in Lemma A.1 of the Appendix.

\subsection{Infeasibility Certificates}

In this section we derive conditions for primal and dual infeasibility. The dual problem associated with problem (1) is

$$
\max _{(x, y)}\left(-\frac{1}{2} x^{T} P x-S_{\mathcal{C}}(y)\right) \quad \text { s.t. } \quad P x+A^{T} y=-q \quad \text { and } \quad y \in\left(\mathcal{C}^{\infty}\right)^{\circ}
$$

and its derivation is included in Lemma A.2 of the Appendix.

We will use the following pair of results to certify infeasibility of (1) in cases where it is primal and/or dual strongly infeasible; we refer the reader to [30] for more details on strong and weak infeasibility.

\section{Proposition 3.1}

(i) If there exists some $\bar{y} \in \mathbb{R}^{m}$ such that

$$
A^{T} \bar{y}=0 \quad \text { and } \quad S_{\mathcal{C}}(\bar{y})<0
$$

then the primal problem (1) is infeasible.

(ii) If there exists some $\bar{x} \in \mathbb{R}^{n}$ such that

$$
P \bar{x}=0, \quad A \bar{x} \in \mathcal{C}^{\infty}, \quad \text { and } \quad\langle q, \bar{x}\rangle<0,
$$

then the dual problem (4) is infeasible. 
Proof (i): The first condition in (5) implies

$$
\inf _{x}\langle\bar{y}, A x\rangle=\inf _{x}\left\langle A^{T} \bar{y}, x\right\rangle=0
$$

and the second condition is equivalent to

$$
\sup _{z \in \mathcal{C}}\langle\bar{y}, z\rangle<0 \text {. }
$$

Therefore, $\left\{z \in \mathbb{R}^{m}:\langle\bar{y}, z\rangle=0\right\}$ is a hyperplane that separates the sets $\left\{A x: x \in \mathbb{R}^{n}\right\}$ and $\mathcal{C}$ strongly [31, Thm. 11.1], meaning that problem (1) is infeasible.

(ii): Define the set $\mathcal{Q}:=\left\{P x+A^{T} y:(x, y) \in \mathbb{R}^{n} \times\left(\mathcal{C}^{\infty}\right)^{\circ}\right\}$. The first two conditions in (6) imply

$$
\begin{aligned}
\sup _{s \in \mathcal{Q}}\langle\bar{x}, s\rangle & =\sup \left\{\left\langle\bar{x}, P x+A^{T} y\right\rangle: x \in \mathbb{R}^{n}, y \in\left(\mathcal{C}^{\infty}\right)^{\circ}\right\} \\
& =\sup _{x}\langle P \bar{x}, x\rangle+\sup \left\{\langle A \bar{x}, y\rangle: y \in\left(\mathcal{C}^{\infty}\right)^{\circ}\right\} \\
& \leq 0
\end{aligned}
$$

where we used the fact that the inner product between vectors in a cone and its polar is non-positive. Since the third condition in (6) can be written as $\langle\bar{x},-q\rangle>0$, this means that $\left\{x \in \mathbb{R}^{n}:\langle\bar{x}, x\rangle=0\right\}$ is a hyperplane that separates the sets $\mathcal{Q}$ and $\{-q\}$ strongly, and thus the dual problem (4) is infeasible.

Note that, if condition (5) in Proposition 3.1 holds, then $\bar{y}$ also represents an unbounded direction in the dual problem assuming it is feasible. Likewise, $\bar{x}$ in condition (6) represents an unbounded direction for the primal problem if it is feasible. However, since we cannot exclude the possibility of simultaneous primal and dual infeasibility, we will refer to condition (5) as primal infeasibility rather than dual unboundedness, and vice versa for (6).

In some cases, e.g., when $\mathcal{C}$ is compact or polyhedral, conditions (5) and (6) in Proposition 3.1 are also necessary for infeasibility, and we say that (5) and (6) are strong alternatives for primal and dual feasibility, respectively. When $\mathcal{C}$ is a convex cone, additional assumptions are required for having strong alternatives; see, e.g., $[32, \S 5.9 .4]$.

Remark 3.1 Due to Assumption 3.1, the support function of $\mathcal{C}$ takes the following form:

$$
S_{\mathcal{C}}(\bar{y})=S_{\mathcal{B}}\left(\bar{y}_{1}\right)+S_{\mathcal{K}_{b}}\left(\bar{y}_{2}\right),
$$

where $\bar{y}=\left(\bar{y}_{1}, \bar{y}_{2}\right)$ with $\bar{y}_{1} \in \mathbb{R}^{m_{1}}$ and $\bar{y}_{2} \in \mathbb{R}^{m_{2}}$. Since the support function of $\mathcal{K}_{b}$ is

$$
S_{\mathcal{K}_{b}}\left(\bar{y}_{2}\right)= \begin{cases}\left\langle b, \bar{y}_{2}\right\rangle, & \bar{y}_{2} \in \mathcal{K}^{\circ}, \\ +\infty, & \text { otherwise }\end{cases}
$$

condition (5) is then equivalent to

$$
A^{T} \bar{y}=0, \quad \bar{y}_{2} \in \mathcal{K}^{\circ}, \quad \text { and } \quad S_{\mathcal{B}}\left(\bar{y}_{1}\right)+\left\langle b, \bar{y}_{2}\right\rangle<0 .
$$




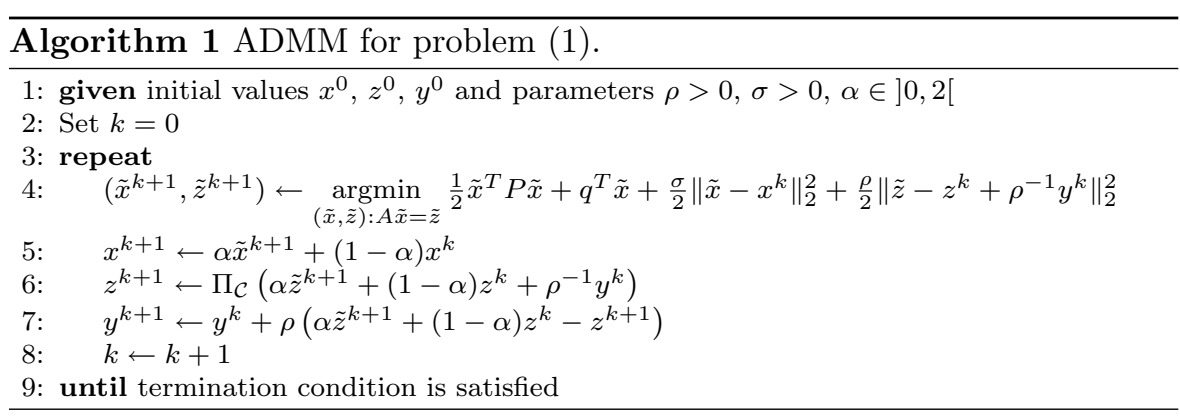

\section{Alternating Direction Method of Multipliers (ADMM)}

ADMM is an operator splitting method that can be used for solving composite minimization problems of the form

$$
\min _{w \in \mathbb{R}^{p}}(f(w)+g(w)),
$$

where $f: \mathbb{R}^{p} \mapsto \tilde{\mathbb{R}}$ and $g: \mathbb{R}^{p} \mapsto \tilde{\mathbb{R}}$ are convex, closed, and proper functions [13]. The iterates of ADMM in application to problem (8) can be written as

$$
\begin{aligned}
& \tilde{w}^{k+1} \leftarrow \operatorname{prox}_{f}\left(w^{k}-u^{k}\right) \\
& w^{k+1} \leftarrow \operatorname{prox}_{g}\left(\alpha \tilde{w}^{k+1}+(1-\alpha) w^{k}+u^{k}\right) \\
& u^{k+1} \leftarrow u^{k}+\alpha \tilde{w}^{k+1}+(1-\alpha) w^{k}-w^{k+1},
\end{aligned}
$$

where $\alpha \in] 0,2[$ is the relaxation parameter.

We can write problem (2) in the general form (8) by setting

$$
\begin{aligned}
& f(x, z)=\frac{1}{2} x^{T} P x+q^{T} x+\mathcal{I}_{A x=z}(x, z), \\
& g(x, z)=\mathcal{I}_{\mathcal{C}}(z) .
\end{aligned}
$$

If we use the norm $\|(x, z)\|=\sqrt{\sigma\|x\|_{2}^{2}+\rho\|z\|_{2}^{2}}$ with $(\sigma, \rho)>0$ in the proximal operators of functions $f$ and $g$, then ADMM reduces to Algorithm 1, which was first introduced in [28]. The scalars $\sigma$ and $\rho$ are called the penalty parameters. Note that the strict positivity of both $\sigma$ and $\rho$ ensure that the equality constrained QP in step 4 of Algorithm 1 has a unique solution for any $P \in \mathbb{S}_{+}^{n}$ and $A \in \mathbb{R}^{m \times n}$.

Unless otherwise stated, we will use $\langle\cdot, \cdot\rangle$ to denote the standard inner product in the Euclidean space, and $\|\cdot\|$ to denote the induced norm. The dimension of the space will be clear from the context.

\subsection{Reformulation as the Douglas-Rachford Splitting (DRS)}

It is well-known that ADMM and DRS are equivalent methods [33]. The authors in [34] show that the ADMM algorithm can be described alternatively in 
terms of the fixed-point iteration of the Douglas-Rachford operator, which is known to be averaged [35]. In particular, the algorithm given by iteration (9) can alternatively be implemented as

$$
\begin{aligned}
w^{k} & \leftarrow \operatorname{prox}_{g}\left(s^{k}\right) \\
\tilde{w}^{k} & \leftarrow \operatorname{prox}_{f}\left(2 w^{k}-s^{k}\right) \\
s^{k+1} & \leftarrow s^{k}+\alpha\left(\tilde{w}^{k}-w^{k}\right) .
\end{aligned}
$$

Similarly, an iteration of Algorithm 1 is equivalent to

$$
\begin{aligned}
\left(\tilde{x}^{k}, \tilde{z}^{k}\right) & \leftarrow \underset{(\tilde{x}, \tilde{z}): A \tilde{x}=\tilde{z}}{\operatorname{argmin}} \frac{1}{2} \tilde{x}^{T} P \tilde{x}+q^{T} \tilde{x}+\frac{\sigma}{2}\left\|\tilde{x}-x^{k}\right\|^{2}+\frac{\rho}{2}\left\|\tilde{z}-\left(2 \Pi_{\mathcal{C}}-\mathrm{Id}\right)\left(v^{k}\right)\right\|^{2} \\
x^{k+1} & \leftarrow x^{k}+\alpha\left(\tilde{x}^{k}-x^{k}\right) \\
v^{k+1} & \leftarrow v^{k}+\alpha\left(\tilde{z}^{k}-\Pi_{\mathcal{C}}\left(v^{k}\right)\right)
\end{aligned}
$$

where

$$
\begin{aligned}
z^{k} & =\Pi_{\mathcal{C}}\left(v^{k}\right) \\
y^{k} & =\rho\left(\operatorname{Id}-\Pi_{\mathcal{C}}\right)\left(v^{k}\right) .
\end{aligned}
$$

We will exploit the following result in the next section to analyze the asymptotic behavior of the algorithm.

Fact 4.1 The iteration described in (12) amounts to

$$
\left(x^{k+1}, v^{k+1}\right) \leftarrow T\left(x^{k}, v^{k}\right),
$$

where $T: \mathbb{R}^{n+m} \mapsto \mathbb{R}^{n+m}$ is an $(\alpha / 2)$-averaged operator.

Proof Iteration (11) is a special case of iteration (49)-(51) in $[34, \S I V-C]$ with $A=\mathrm{Id}, B=-\mathrm{Id}$ and $c=0$, which is equivalent to

$$
s^{k+1} \leftarrow T_{\mathrm{DR}} s^{k}
$$

where $T_{D R}$ is the Douglas-Rachford operator given by

$$
T_{\mathrm{DR}}=\left(1-\frac{\alpha}{2}\right) \mathrm{Id}+\frac{\alpha}{2}\left(2 \operatorname{prox}_{f}-\mathrm{Id}\right) \circ\left(2 \operatorname{prox}_{g}-\mathrm{Id}\right),
$$

which is known to be $(\alpha / 2)$-averaged $[17,35]$. The result follows from the fact that iteration (12) is a special case of iteration (11) with $f$ and $g$ given by (10), and the inner product given by $\left\langle\left(x_{1}, z_{1}\right),\left(x_{2}, z_{2}\right)\right\rangle=\sigma\left\langle x_{1}, x_{2}\right\rangle+\rho\left\langle z_{1}, z_{2}\right\rangle$.

Due to [15, Prop. 6.46], the identities in (13) imply that in each iteration the pair $\left(z^{k}, y^{k}\right)$ satisfies optimality condition (3c) by construction. The solution to the equality constrained QP in (12a) satisfies the pair of optimality conditions

$$
\begin{aligned}
& 0=A \tilde{x}^{k}-\tilde{z}^{k} \\
& 0=(P+\sigma I) \tilde{x}^{k}+q-\sigma x^{k}+\rho A^{T}\left(\tilde{z}^{k}-\left(2 \Pi_{\mathcal{C}}-\mathrm{Id}\right)\left(v^{k}\right)\right) .
\end{aligned}
$$


If we rearrange $(12 \mathrm{~b})$ and $(12 \mathrm{c})$ to isolate $\tilde{x}^{k}$ and $\tilde{z}^{k}$, i.e., write

$$
\begin{aligned}
& \tilde{x}^{k}=x^{k}+\alpha^{-1} \delta x^{k+1} \\
& \tilde{z}^{k}=z^{k}+\alpha^{-1} \delta v^{k+1},
\end{aligned}
$$

and substitute them into (14), then using (13) we obtain the following relations between the iterates:

$$
\begin{aligned}
A x^{k}-\Pi_{\mathcal{C}}\left(v^{k}\right) & =-\alpha^{-1}\left(A \delta x^{k+1}-\delta v^{k+1}\right) \\
P x^{k}+q+\rho A^{T}\left(\operatorname{Id}-\Pi_{\mathcal{C}}\right)\left(v^{k}\right) & =-\alpha^{-1}\left((P+\sigma I) \delta x^{k+1}+\rho A^{T} \delta v^{k+1}\right) .
\end{aligned}
$$

Observe that the right-hand terms of (16) are a direct measure of how far the iterates $\left(x^{k}, z^{k}, y^{k}\right)$ are from satisfying optimality conditions (3a) and (3b). We refer to the left-hand terms of (16a) and (16b) as the primal and dual residuals, respectively. In the next section, we will show that the successive differences $\left(\delta x^{k}, \delta v^{k}\right)$ appearing in the right-hand side of (16) converge and can be used to test for primal and dual infeasibility.

\section{Asymptotic Behavior of ADMM}

In order to analyze the asymptotic behavior of iteration (12), which is equivalent to Algorithm 1, we will rely heavily on the following results:

Lemma 5.1 Let $\mathcal{D}$ be a nonempty, closed, and convex subset of $\mathbb{R}^{n}$ and suppose that $T: \mathcal{D} \mapsto \mathcal{D}$ is an averaged operator. Let $s^{0} \in \mathcal{D}, s^{k}=T^{k} s^{0}$, and $\delta s$ be the projection of the zero vector onto $\mathrm{cl} \operatorname{ran}(T-\mathrm{Id})$. Then

(i) $\frac{1}{k} s^{k} \rightarrow \delta s$.

(ii) $\delta s^{k} \rightarrow \delta s$.

(iii) If $\operatorname{Fix} T \neq \emptyset$, then $\left\{s^{k}\right\}_{k \in \mathbb{N}}$ converges to a point in $\operatorname{Fix} T$.

Proof The first result is [36, Cor. 3], the second is [37, Cor. 2.3], and the third is [15, Thm. 5.14].

Note that, since $\operatorname{ran}(T-\mathrm{Id})$ is not necessarily closed or convex, the projection onto this set may not exist, but the projection onto its closure always exists. Moreover, since clran( $T-\mathrm{Id})$ is convex [36, Lem. 4], the projection is unique. Due to Fact 4.1, Lemma 5.1 ensures that $\left(\frac{1}{k} x^{k}, \frac{1}{k} v^{k}\right) \rightarrow(\delta x, \delta v)$ and $\left(\delta x^{k}, \delta v^{k}\right) \rightarrow(\delta x, \delta v)$.

The core results of this paper are contained within the following two propositions, which establish various relationships between the limits $\delta x$ and $\delta v$; we include several supporting results required to prove these results in the Appendix. Given these two results, it will then be straightforward to extract certificates of optimality or infeasibility in Section 5.1. For both of these central results, and in the remainder of the paper, we define

$$
\begin{aligned}
\delta z & :=\Pi_{\mathcal{C}^{\infty}}(\delta v) \\
\delta y & :=\rho \Pi_{\left(\mathcal{C}^{\infty}\right)^{\circ}}(\delta v) .
\end{aligned}
$$


Proposition 5.1 Suppose that Assumption 3.1 holds. Then the following relations hold between the limits $\delta x, \delta z$, and $\delta y$ :
(i) $A \delta x=\delta z$
(ii) $P \delta x=0$.
(iii) $A^{T} \delta y=0$.
(iv) $\frac{1}{k} z^{k} \rightarrow \delta z$ and $\delta z^{k} \rightarrow \delta z$.
(v) $\frac{1}{k} y^{k} \rightarrow \delta y$ and $\delta y^{k} \rightarrow \delta y$

Proof Commensurate with our partitioning of the constraint set as $\mathcal{C}=\mathcal{B} \times \mathcal{K}_{b}$, we partition the matrix $A$ and the iterates into components of appropriate dimension. We use subscript 1 for those components associated with the set $\mathcal{B}$ and subscript 2 for those associated with the set $\mathcal{K}_{b}$, e.g., $z^{k}=\left(z_{1}^{k}, z_{2}^{k}\right)$ where $z_{1}^{k} \in \mathcal{B}$ and $z_{2}^{k} \in \mathcal{K}_{b}$, and the matrix $A=\left[A_{1} ; A_{2}\right]$. Note throughout that $\mathcal{C}^{\infty}=\{0\} \times \mathcal{K}$ and $\left(\mathcal{C}^{\infty}\right)^{\circ}=\mathbb{R}^{m_{1}} \times \mathcal{K}^{\circ}$, and thus

$$
\begin{aligned}
\Pi_{\mathcal{C}^{\infty}}(\delta v) & =\left[\begin{array}{c}
0 \\
\Pi_{\mathcal{K}}\left(\delta v_{2}\right)
\end{array}\right] \\
\Pi_{\left(\mathcal{C}^{\infty}\right)}(\delta v) & =\left[\begin{array}{c}
\delta v_{1} \\
\Pi_{\mathcal{K}^{\circ}}\left(\delta v_{2}\right)
\end{array}\right] .
\end{aligned}
$$

(i): Divide (16a) by $k$, take the limit, and apply Lemma 5.1 to get

$$
A \delta x=\lim _{k \rightarrow \infty} \frac{1}{k} \Pi_{\mathcal{C}}\left(v^{k}\right) .
$$

Due to Lemma A.4 and the compactness of $\mathcal{B}$, we have

$$
\left[\begin{array}{l}
A_{1} \delta x \\
A_{2} \delta x
\end{array}\right]=\lim _{k \rightarrow \infty}\left[\begin{array}{c}
\frac{1}{k} \Pi_{\mathcal{B}}\left(v_{1}^{k}\right) \\
\frac{1}{k} \Pi_{\mathcal{K}_{b}}\left(v_{2}^{k}\right)
\end{array}\right]=\left[\begin{array}{c}
0 \\
\Pi_{\mathcal{K}}\left(\delta v_{2}\right)
\end{array}\right] .
$$

Combining the equalities above with (18a) and (17a), we obtain

$$
A \delta x=\lim _{k \rightarrow \infty} \frac{1}{k} \Pi_{\mathcal{C}}\left(v^{k}\right)=\Pi_{\mathcal{C} \infty}(\delta v)=\delta z .
$$

(ii): Divide (16b) by $\rho k$, take the inner product of both sides with $\delta x$ and take the limit to obtain

$$
\begin{aligned}
-\rho^{-1}\langle P \delta x, \delta x\rangle & =\lim _{k \rightarrow \infty}\left\langle A \delta x, \frac{1}{k} v_{k}-\frac{1}{k} \Pi_{\mathcal{C}}\left(v^{k}\right)\right\rangle \\
& =\left\langle\Pi_{\mathcal{C} \infty}(\delta v), \delta v-\Pi_{\mathcal{C}}(\delta v)\right\rangle \\
& =\left\langle\Pi_{\mathcal{C} \infty}(\delta v), \Pi_{\left(\mathcal{C}^{\infty}\right)}(\delta v)\right\rangle \\
& =0
\end{aligned}
$$

where we used Lemma 5.1 and (20) in the second equality, and the Moreau decomposition [15, Thm. 6.29] in the third and fourth. Since $P \in \mathbb{S}_{+}^{n}$, it follows that

$$
P \delta x=0
$$


(iii): Divide (16b) by $k$, take the limit, and use (21) to obtain

$$
\begin{aligned}
0 & =\lim _{k \rightarrow \infty} \frac{1}{k} \rho A^{T}\left(\operatorname{Id}-\Pi_{\mathcal{C}}\right)\left(v^{k}\right) \\
& =\rho A^{T} \lim _{k \rightarrow \infty}\left(\frac{1}{k} v^{k}-\frac{1}{k} \Pi_{\mathcal{C}}\left(v^{k}\right)\right) \\
& =\rho A^{T}\left(\delta v-\Pi_{\mathcal{C} \infty}(\delta v)\right) \\
& =A^{T} \rho \Pi_{\left(\mathcal{C}^{\infty}\right)^{\circ}}(\delta v) \\
& =A^{T} \delta y,
\end{aligned}
$$

where we used Lemma 5.1 and (20) in the third equality, the Moreau decomposition in the fourth, and (17b) in the fifth.

(iv): We first show that the sequence $\left\{\delta z^{k}\right\}_{k \in \mathbb{N}}$ converges to $\delta z$. From (15) we have

$$
\begin{aligned}
& -\alpha^{-1}\left(\delta x^{k+1}-\delta x^{k}\right)=\delta x^{k}-\delta \tilde{x}^{k} \\
& -\alpha^{-1}\left(\delta v^{k+1}-\delta v^{k}\right)=\delta z^{k}-\delta \tilde{z}^{k}
\end{aligned}
$$

Take the limit of (22a) to obtain

$$
\lim _{k \rightarrow \infty} \delta \tilde{x}^{k}=\lim _{k \rightarrow \infty} \delta x^{k}=\delta x .
$$

From (14a) we now have $\delta \tilde{z}^{k}=A \delta \tilde{x}^{k} \rightarrow A \delta x$. Take the limit of (22b) and use (20) to obtain

$$
\lim _{k \rightarrow \infty} \delta z^{k}=\lim _{k \rightarrow \infty} \delta \tilde{z}^{k}=A \delta x=\delta z .
$$

We now show that the sequence $\left\{\frac{1}{k} z^{k}\right\}_{k \in \mathbb{N}}$ also converges to $\delta z$. Dividing (13a) by $k$ and taking the limit, we obtain

$$
\lim _{k \rightarrow \infty} \frac{1}{k} z^{k}=\lim _{k \rightarrow \infty} \frac{1}{k} \Pi_{\mathcal{C}}\left(v^{k}\right)=\delta z,
$$

where the second equality follows from (20).

$(\mathrm{v})$ : We first show that the sequence $\left\{\delta y^{k}\right\}_{k \in \mathbb{N}}$ converges to $\delta y$. From (13) we have $y^{k}=\rho\left(v^{k}-z^{k}\right)$, and thus

$$
\lim _{k \rightarrow \infty} \delta y^{k}=\rho \lim _{k \rightarrow \infty}\left(\delta v^{k}-\delta z^{k}\right)=\rho\left(\delta v-\Pi_{\mathcal{C} \infty}(\delta v)\right)=\rho \Pi_{\left(\mathcal{C}^{\infty}\right)^{\circ}}(\delta v)=\delta y,
$$

where we used the Moreau decomposition in the third equality, and (17b) in the last.

We now show that the sequence $\left\{\frac{1}{k} y^{k}\right\}_{k \in \mathbb{N}}$ also converges to $\delta y$. Dividing (13b) by $k$ and taking the limit, we obtain

$$
\lim _{k \rightarrow \infty} \frac{1}{k} y^{k}=\rho \lim _{k \rightarrow \infty}\left(\frac{1}{k} v^{k}-\frac{1}{k} \Pi_{\mathcal{C}}\left(v^{k}\right)\right)=\rho\left(\delta v-\Pi_{\mathcal{C} \infty}(\delta v)\right)=\delta y .
$$

Proposition 5.1 shows that the limits $\delta y$ and $\delta x$ will always satisfy the subspace and conic constraints in the primal and dual infeasibility conditions (5) and (6), respectively. We next consider the terms appearing in the inequalities in (5) and (6). 
Proposition 5.2 Suppose that Assumption 3.1 holds. Then the following identities hold for the limits $\delta x$ and $\delta y$ :

(i) $\langle q, \delta x\rangle=-\sigma \alpha^{-1}\|\delta x\|^{2}-\rho \alpha^{-1}\|A \delta x\|^{2}$.

(ii) $S_{\mathcal{C}}(\delta y)=-\rho^{-1} \alpha^{-1}\|\delta y\|^{2}$.

Proof Take the inner product of both sides of (16b) with $\delta x$ and use Proposition 5.1(ii) to obtain

$$
\langle q, \delta x\rangle+\rho\left\langle A \delta x,\left(\operatorname{Id}-\Pi_{\mathcal{C}}\right)\left(v^{k}\right)\right\rangle=-\sigma \alpha^{-1}\left\langle\delta x, \delta x^{k+1}\right\rangle-\rho \alpha^{-1}\left\langle A \delta x, \delta v^{k+1}\right\rangle .
$$

Using (19) and then taking the limit gives

$$
\begin{aligned}
\langle q, \delta x\rangle= & -\sigma \alpha^{-1}\|\delta x\|^{2}-\rho \alpha^{-1}\langle A \delta x, \delta v\rangle \\
& -\rho \lim _{k \rightarrow \infty}\left\langle\Pi_{\mathcal{K}}\left(\delta v_{2}\right), \Pi_{\mathcal{K}^{\circ}}\left(v_{2}^{k}-b\right)\right\rangle \\
= & -\sigma \alpha^{-1}\|\delta x\|^{2}-\rho \alpha^{-1}\left\langle\Pi_{\mathcal{C}^{\infty}}(\delta v), \delta v\right\rangle \\
& -\rho \lim _{k \rightarrow \infty}\left\langle\Pi_{\mathcal{K}}\left(\delta v_{2}\right), \Pi_{\mathcal{K}^{\circ}}\left(v_{2}^{k}-b\right)\right\rangle \\
= & -\sigma \alpha^{-1}\|\delta x\|^{2}-\rho \alpha^{-1}\left\|\Pi_{\mathcal{C}^{\infty}}(\delta v)\right\|^{2} \\
& -\rho \lim _{k \rightarrow \infty}\left\langle\Pi_{\mathcal{K}}\left(\delta v_{2}\right), \Pi_{\mathcal{K}^{\circ}}\left(v_{2}^{k}-b\right)\right\rangle,
\end{aligned}
$$

where we used Lemma A.3(ii) in the first equality, (20) in the second, and Lemma A.3(iv) in the third.

Now take the inner product of both sides of $(16 \mathrm{a})$ with $\Pi_{(\mathcal{C} \infty)^{\circ}}(\delta v)$ to obtain

$$
\begin{aligned}
\alpha^{-1}\left\langle\Pi_{(\mathcal{C} \infty)^{\circ}}(\delta v), \delta v^{k+1}\right\rangle= & \left\langle A^{T} \Pi_{\left(\mathcal{C}^{\infty}\right)^{\circ}}(\delta v), x^{k}+\alpha^{-1} \delta x^{k+1}\right\rangle \\
& -\left\langle\Pi_{\left(\mathcal{C}^{\infty}\right)^{\circ}}(\delta v), \Pi_{\mathcal{C}}\left(v^{k}\right)\right\rangle .
\end{aligned}
$$

According to Proposition 5.1(iii) and (17b), the first inner product on the right-hand side is zero. Taking the limit we obtain

$$
\begin{aligned}
\lim _{k \rightarrow \infty}\left\langle\Pi_{\left(\mathcal{C}^{\infty}\right)^{\circ}}(\delta v), \Pi_{\mathcal{C}}\left(v^{k}\right)\right\rangle & =-\alpha^{-1}\left\langle\Pi_{\left(\mathcal{C}^{\infty}\right)^{\circ}}(\delta v), \delta v\right\rangle \\
& =-\alpha^{-1}\left\|\Pi_{\left(\mathcal{C}^{\infty}\right)^{\circ}}(\delta v)\right\|^{2},
\end{aligned}
$$

where the second equality follows from Lemma A.3(iv). Using (18b), we can write the equality above as

$$
\begin{aligned}
-\alpha^{-1}\left\|\Pi_{(\mathcal{C} \infty)}(\delta v)\right\|^{2}= & \lim _{k \rightarrow \infty}\left\langle\delta v_{1}, \Pi_{\mathcal{B}}\left(v_{1}^{k}\right)\right\rangle+\left\langle\Pi_{\mathcal{K}^{\circ}}\left(\delta v_{2}\right), \Pi_{\mathcal{K}_{b}}\left(v_{2}^{k}\right)\right\rangle \\
= & S_{\mathcal{B}}\left(\delta v_{1}\right)+\left\langle\Pi_{\mathcal{K}^{\circ}}\left(\delta v_{2}\right), b\right\rangle \\
& +\lim _{k \rightarrow \infty}\left\langle\Pi_{\mathcal{K}^{\circ}}\left(\delta v_{2}\right), \Pi_{\mathcal{K}_{b}}\left(v_{2}^{k}-b\right)\right\rangle \\
= & S_{\mathcal{B}}\left(\delta v_{1}\right)+S_{\mathcal{K}_{b}}\left(\Pi_{\mathcal{K}^{\circ}}\left(\delta v_{2}\right)\right) \\
& +\lim _{k \rightarrow \infty}\left\langle\Pi_{\mathcal{K}^{\circ}}\left(\delta v_{2}\right), \Pi_{\mathcal{K}_{b}}\left(v_{2}^{k}-b\right)\right\rangle \\
= & S_{\mathcal{C}}\left(\Pi_{\left(\mathcal{C}^{\infty}\right)^{\circ}}(\delta v)\right)+\lim _{k \rightarrow \infty}\left\langle\Pi_{\mathcal{K}^{\circ}}\left(\delta v_{2}\right), \Pi_{\mathcal{K}_{b}}\left(v_{2}^{k}-b\right)\right\rangle,
\end{aligned}
$$


where the second equality follows from Lemma A.3(i) and Lemma A.5, the third from Lemma A.3(v), and the fourth from (18b). Multiplying by $\rho$ and using (17b) and the positive homogeneity of $S_{\mathcal{C}}$, we obtain

$$
S_{\mathcal{C}}(\delta y)=-\rho \alpha^{-1}\left\|\Pi_{(\mathcal{C} \infty)^{\circ}}(\delta v)\right\|^{2}-\rho \lim _{k \rightarrow \infty}\left\langle\Pi_{\mathcal{K}^{\circ}}\left(\delta v_{2}\right), \Pi_{\mathcal{K}}\left(v_{2}^{k}-b\right)\right\rangle .
$$

We will next show that the limits in (23) and (24) are equal to zero. Summing the two equalities, we obtain

$$
\begin{aligned}
\langle q, \delta x\rangle+S_{\mathcal{C}}(\delta y)+\sigma \alpha^{-1}\|\delta x\|^{2}+ & \rho \alpha^{-1}\|\delta v\|^{2} \\
= & -\rho \lim _{k \rightarrow \infty}\left\langle\Pi_{\mathcal{K}}\left(\delta v_{2}\right), \Pi_{\mathcal{K}^{\circ}}\left(v_{2}^{k}-b\right)\right\rangle \\
& -\rho \lim _{k \rightarrow \infty}\left\langle\Pi_{\mathcal{K}^{\circ}}\left(\delta v_{2}\right), \Pi_{\mathcal{K}}\left(v_{2}^{k}-b\right)\right\rangle,
\end{aligned}
$$

where we used $\|\delta v\|^{2}=\left\|\Pi_{\mathcal{C} \infty}(\delta v)\right\|^{2}+\left\|\Pi_{\left(\mathcal{C}^{\infty}\right)^{\circ}}(\delta v)\right\|^{2}[15$, Thm. 6.29].

Now take the inner product of both sides of (16b) with $x^{k}$ to obtain

$$
\begin{aligned}
\left\langle P x^{k}, x^{k}\right\rangle+\left\langle q, x^{k}\right\rangle+\rho\left\langle A x^{k},\left(\operatorname{Id}-\Pi_{\mathcal{C}}\right)\left(v^{k}\right)\right\rangle= & -\alpha^{-1}\left\langle P \delta x^{k+1}, x^{k}\right\rangle \\
& -\sigma \alpha^{-1}\left\langle\delta x^{k+1}, x^{k}\right\rangle \\
& -\rho \alpha^{-1}\left\langle A x^{k}, \delta v^{k+1}\right\rangle .
\end{aligned}
$$

We can rewrite the third inner product on the left-hand side of (26) as

$$
\begin{aligned}
\left\langle A x^{k},\left(\operatorname{Id}-\Pi_{\mathcal{C}}\right)\left(v^{k}\right)\right\rangle= & \left\langle\Pi_{\mathcal{C}}\left(v^{k}\right)+\alpha^{-1}\left(\delta v^{k+1}-A \delta x^{k+1}\right),\left(\operatorname{Id}-\Pi_{\mathcal{C}}\right)\left(v^{k}\right)\right\rangle \\
= & \left\langle\Pi_{\mathcal{B}}\left(v_{1}^{k}\right), v_{1}^{k}\right\rangle-\left\|\Pi_{\mathcal{B}}\left(v_{1}^{k}\right)\right\|^{2} \\
& +\left\langle\Pi_{\mathcal{K}_{b}}\left(v_{2}^{k}\right),\left(\operatorname{Id}-\Pi_{\mathcal{K}_{b}}\right)\left(v_{2}^{k}\right)\right\rangle \\
& +\alpha^{-1}\left\langle\delta v^{k+1}-A \delta x^{k+1}, \rho^{-1} y^{k}\right\rangle \\
= & \left\langle\Pi_{\mathcal{B}}\left(v_{1}^{k}\right), v_{1}^{k}\right\rangle-\left\|\Pi_{\mathcal{B}}\left(v_{1}^{k}\right)\right\|^{2}+\left\langle b, \Pi_{\mathcal{K}^{\circ}}\left(v_{2}^{k}-b\right)\right\rangle \\
& +\alpha^{-1}\left\langle\delta v^{k+1}-A \delta x^{k+1}, \rho^{-1} y^{k}\right\rangle,
\end{aligned}
$$

where we used (16a) in the first equality, (13b) in the second, and Lemma A.3(iii) in the third. Substituting this expression into (26), dividing by $k$, and taking the limit, we obtain

$$
\begin{aligned}
\lim _{k \rightarrow \infty} \frac{1}{k}\left\langle P x^{k}, x^{k}\right\rangle+\langle q, \delta x\rangle+S_{\mathcal{C}}(\delta y) & +\sigma \alpha^{-1}\|\delta x\|^{2} \\
= & -\rho \alpha^{-1}\left\langle\delta v-A \delta x, \rho^{-1} \delta y\right\rangle \\
& -\rho \alpha^{-1}\langle A \delta x, \delta v\rangle,
\end{aligned}
$$

where we used Lemma A.3(v), Lemma A.4, Lemma A.5, Proposition 5.1(ii), $(17 \mathrm{~b}),(18 \mathrm{~b})$, and the compactness of $\mathcal{B}$. The sum of inner products appearing on the right-hand side of (27) can be written as

$$
\begin{aligned}
\left\langle\delta v-A \delta x, \rho^{-1} \delta y\right\rangle+\langle A \delta x, \delta v\rangle & =\left\langle\delta v-\Pi_{\mathcal{C}^{\infty}}(\delta v), \Pi_{\left(\mathcal{C}^{\infty}\right)^{\circ}}(\delta v)\right\rangle+\left\langle\Pi_{\mathcal{C}^{\infty}}(\delta v), \delta v\right\rangle \\
& =\left\|\Pi_{(\mathcal{C} \infty)^{\circ}}(\delta v)\right\|^{2}+\left\|\Pi_{\mathcal{C}^{\infty}}(\delta v)\right\|^{2} \\
& =\|\delta v\|^{2}
\end{aligned}
$$


where we used (17b) and (20) in the first equality, and Lemma A.3(iv) and the Moreau decomposition in the second. Substituting the equality above into (27), we obtain

$$
\langle q, \delta x\rangle+S_{\mathcal{C}}(\delta y)+\sigma \alpha^{-1}\|\delta x\|^{2}+\rho \alpha^{-1}\|\delta v\|^{2}=-\lim _{k \rightarrow \infty} \frac{1}{k}\left\langle P x^{k}, x^{k}\right\rangle .
$$

Comparing the identities in (25) and (28), we get the following relation:

$$
\begin{aligned}
\lim _{k \rightarrow \infty} \frac{1}{k}\left\langle P x^{k}, x^{k}\right\rangle= & \rho \lim _{k \rightarrow \infty}\left\langle\Pi_{\mathcal{K}}\left(\delta v_{2}\right), \Pi_{\mathcal{K}^{\circ}}\left(v_{2}^{k}-b\right)\right\rangle \\
& +\rho \lim _{k \rightarrow \infty}\left\langle\Pi_{\mathcal{K}^{\circ}}\left(\delta v_{2}\right), \Pi_{\mathcal{K}}\left(v_{2}^{k}-b\right)\right\rangle .
\end{aligned}
$$

The positive semidefiniteness of $P$ implies that the sequence on the left-hand side is term-wise non-negative. Since the two sequences on the right-hand side involve inner products of elements in $\mathcal{K}$ and $\mathcal{K}^{\circ}$, each sequence is term-wise non-positive. Consequently, each of these limits must be zero. Finally, using (17b) and (20), the claims of the proposition then follow directly from (23) and (24)

\subsection{Optimality and Infeasibility Certificates}

We are now in a position to prove that, in the limit, the iterates of Algorithm 1 either satisfy the optimality conditions (3) or produce a certificate of strong infeasibility. Recall that Fact 4.1, Lemma 5.1(ii), and Proposition 5.1(iv)-(v) ensure convergence of the sequence $\left\{\delta x^{k}, \delta z^{k}, \delta y^{k}\right\}_{k \in \mathbb{N}}$.

Proposition 5.3 (Optimality) If $\left(\delta x^{k}, \delta z^{k}, \delta y^{k}\right) \rightarrow(0,0,0)$, then the optimality conditions (3) are satisfied in the limit, i.e.,

$$
\left\|P x^{k}+q+A^{T} y^{k}\right\| \rightarrow 0 \text { and }\left\|A x^{k}-z^{k}\right\| \rightarrow 0 .
$$

Proof Follows from (13) and (16).

Lemma 5.1(iii) is sufficient to prove that, if problem (1) is solvable, then the sequence of iterates $\left\{x^{k}, z^{k}, y^{k}\right\}_{k \in \mathbb{N}}$ converges to its primal-dual solution. However, convergence of $\left\{\delta x^{k}, \delta z^{k}, \delta y^{k}\right\}_{k \in \mathbb{N}}$ to zero is not itself sufficient to prove convergence of $\left\{x^{k}, z^{k}, y^{k}\right\}_{k \in \mathbb{N}}$; we provide a numerical example in Section 6.3 to show when this scenario can occur. According to Proposition 5.3, in this case the violation of optimality conditions still goes to zero in the limit.

We next show that, if $\left\{\delta x^{k}, \delta z^{k}, \delta y^{k}\right\}_{k \in \mathbb{N}}$ converges to a nonzero value, then we can construct a certificate of primal and/or dual infeasibility. Note that, due to Proposition 5.1(i), $\delta z$ can be nonzero only when $\delta x$ is nonzero.

Theorem 5.1 (Infeasibility) Suppose that Assumption 3.1 holds.

(i) If $\delta y \neq 0$, then problem (1) is infeasible and $\delta y$ satisfies the primal infeasibility condition (5). 
(ii) If $\delta x \neq 0$, then problem (4) is infeasible and $\delta x$ satisfies the dual infeasibility condition (6).

(iii) If $\delta x \neq 0$ and $\delta y \neq 0$, then problems (1) and (4) are simultaneously infeasible.

Proof (i): Follows from Proposition 5.1(iii) and Proposition 5.2(ii).

(ii): Follows from Proposition 5.1(i)-(ii) and Proposition 5.2(i).

(iii): Follows from (i) and (ii).

Remark 5.1 It is easy to show that $\delta y$ and $\delta x$ would still provide certificates of primal and dual infeasibility if we instead used the norm $\|(x, z)\|=\sqrt{x^{T} S x+z^{T} R z}$ in the proximal operators in (9), with $R$ and $S$ being diagonal positive definite matrices.

\subsection{Termination Criteria}

We can define termination criteria for Algorithm 1 so that the iterations stop when either a primal-dual solution or a certificate of primal or dual infeasibility is found with some predefined accuracy.

A reasonable criterion for detecting optimality is that the norms of primal and dual residuals are smaller than some tolerance levels $\varepsilon_{\text {prim }}>0$ and $\varepsilon_{\text {dual }}>0$, respectively, i.e.,

$$
\left\|A x^{k}-z^{k}\right\| \leq \varepsilon_{\text {prim }}, \quad\left\|P x^{k}+q+A^{T} y^{k}\right\| \leq \varepsilon_{\text {dual }} .
$$

Since $\left(\delta x^{k}, \delta y^{k}\right) \rightarrow(\delta x, \delta y)$, a meaningful criterion for detecting primal and dual infeasibility would be to use $\delta y^{k}$ and $\delta x^{k}$ to check that conditions (7) and (6) are almost satisfied, i.e.,

$$
\left\|A^{T} \delta y^{k}\right\| \leq \varepsilon_{\text {pinf }}, \quad \operatorname{dist}_{\mathcal{K}^{\circ}}\left(\delta y_{2}^{k}\right) \leq \varepsilon_{\text {pinf }}, \quad S_{\mathcal{B}}\left(\delta y_{1}^{k}\right)+\left\langle b, \delta y_{2}^{k}\right\rangle<\varepsilon_{\text {pinf }},
$$

and

$$
\left\|P \delta x^{k}\right\| \leq \varepsilon_{\text {dinf }}, \quad \operatorname{dist}_{\mathcal{C}^{\infty}}\left(A \delta x^{k}\right) \leq \varepsilon_{\operatorname{dinf}}, \quad\left\langle q, \delta x^{k}\right\rangle<\varepsilon_{\operatorname{dinf}},
$$

where $\varepsilon_{\text {pinf }}>0$ and $\varepsilon_{\text {dinf }}>0$. Infeasibility detection based on these vectors is used in OSQP [28], an open-source operator splitting solver for quadratic programming. Note that the tolerance levels are often chosen relative to the scaling of the algorithm's iterates and the problem data; see [28, Sec. 3.4] for details.

Although the optimality or infeasiblity conditions are guaranteed to be satisfied exactly only in the limit, at least one of the termination criteria given by (29)-(31) will be satisfied after finitely many iterations for any positive tolerance levels $\varepsilon_{\text {prim }}>0, \varepsilon_{\text {dual }}>0, \varepsilon_{\text {pinf }}>0$, and $\varepsilon_{\text {dinf }}>0$. For weakly infeasible problems termination criteria for both optimality and infeasibility will be satisfied for any given accuracy. This means that an infinitesimally small perturbation to the problem can make it solvable or strongly infeasible. We provide an example in Section 6.3 illustrating such case. 
Remark 5.2 Even though $\left(\delta x^{k}, \delta y^{k}\right) \rightarrow(\delta x, \delta y)$, termination criteria for detecting infeasibility should not be implemented by simply checking that successive terms in the sequences $\left\{\delta x^{k}\right\}_{k \in \mathbb{N}}$ and $\left\{\delta y^{k}\right\}_{k \in \mathbb{N}}$ are close together. The reason is that these sequences can take values which repeat for many iterations even though they have not reached their limit points, and such repeated values in these sequences will not necessarily constitute infeasibility certificates. Instead, we check the infeasibility conditions (30) and (31) directly, with the understanding that these conditions will necessarily be satisfied in the limit for infeasible problems.

Remark 5.3 Instead of using $\delta y^{k}$ in the primal infeasibility criterion (30), we could instead use the vector

$$
\Pi_{\left(\mathcal{C}^{\infty}\right)^{\circ}}\left(\delta y^{k}\right)=\left[\begin{array}{c}
\delta y_{1}^{k} \\
\Pi_{\mathcal{K}^{\circ}}\left(\delta y_{2}^{k}\right)
\end{array}\right] .
$$

Note that the second condition in (30) would then be satisfied by construction.

\section{Numerical Examples}

In this section, we demonstrate via several numerical examples the different asymptotic behaviors of the iterates generated by Algorithm 1 for solving optimization problems of the form (1).

\subsection{Parametric QP}

Consider the QP

$$
\begin{aligned}
\min _{\left(x_{1}, x_{2}\right)} & \left(\frac{1}{2} x_{1}^{2}+x_{1}-x_{2}\right) \\
\text { s.t. } & 0 \leq x_{1}+a x_{2} \leq u_{1} \\
& 1 \leq x_{1} \leq 3 \\
& 1 \leq x_{2} \leq u_{3},
\end{aligned}
$$

where $a \in \mathbb{R}, u_{1} \geq 0$, and $u_{3} \geq 1$ are parameters. Note that the problem above is an instance of problem (1) with

$$
P=\left[\begin{array}{ll}
1 & 0 \\
0 & 0
\end{array}\right], \quad q=\left[\begin{array}{c}
1 \\
-1
\end{array}\right], \quad A=\left[\begin{array}{ll}
1 & a \\
1 & 0 \\
0 & 1
\end{array}\right], \quad \mathcal{C}=[l, u], \quad l=\left[\begin{array}{l}
0 \\
1 \\
1
\end{array}\right], \quad u=\left[\begin{array}{c}
u_{1} \\
3 \\
u_{3}
\end{array}\right]
$$

where $[l, u]:=\left\{z \in \mathbb{R}^{m}: l \leq z \leq u\right\}$. Depending on the values of parameters $u_{1}$ and $u_{3}$, the constraint set in (32) can be either bounded or unbounded. The projection onto the set $[l, u]$ can be evaluated as

$$
\Pi_{[l, u]}(z)=\max (\min (z, u), l),
$$

and the support function of the bounded set $\mathcal{B}=[l, u]$ as

$$
S_{\mathcal{B}}(y)=\langle l, \min (y, 0)\rangle+\langle u, \max (y, 0)\rangle,
$$




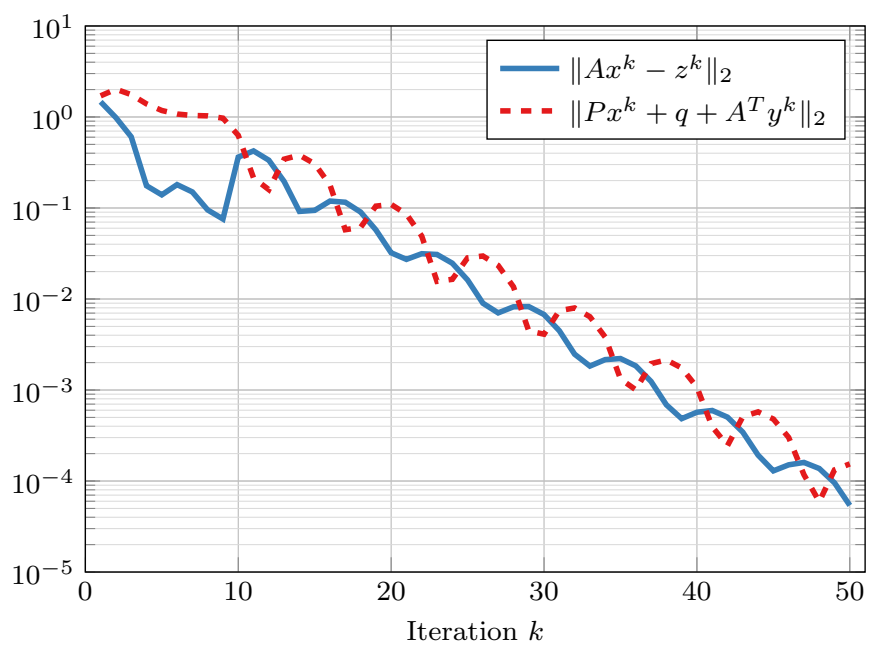

Fig. 1 Convergence of $\left\{x^{k}, z^{k}, y^{k}\right\}_{k \in \mathbb{N}}$ to a certificate of optimality for problem (32) with $a=1, u_{1}=5$ and $u_{3}=3$.

where min and max functions should be taken element-wise.

In the sequel we will discuss four scenarios that can occur depending on the values of the parameters: (i) optimality, (ii) primal infeasibility, (iii) dual infeasibility, (iv) simultaneous primal and dual infeasibility, and will show that Algorithm 1 correctly produces certificates for all four scenarios. In all cases we set the parameters $\alpha=\rho=\sigma=1$ and set the initial iterate $\left(x^{0}, z^{0}, y^{0}\right)=(0,0,0)$.

Optimality.

Consider problem (32) with parameters

$$
a=1, \quad u_{1}=5, \quad u_{3}=3 .
$$

Algorithm 1 converges to $x^{\star}=(1,3), z^{\star}=(4,1,3), y^{\star}=(0,-2,1)$, for which the objective value equals -1.5 , and we have

$$
A x^{\star}-z^{\star}=0 \text { and } P x^{\star}+q+A^{T} y^{\star}=0,
$$

i.e., the pair $\left(x^{\star}, y^{\star}\right)$ is a primal-dual solution to problem (32). Figure 1 shows convergence of $\left\{x^{k}, z^{k}, y^{k}\right\}_{k \in \mathbb{N}}$ to a certificate of optimality. Recall that the iterates of the algorithm always satisfy the optimality conditions (3c).

Primal infeasibility.

We next set the parameters of problem (32) to

$$
a=1, \quad u_{1}=0, \quad u_{3}=3 .
$$



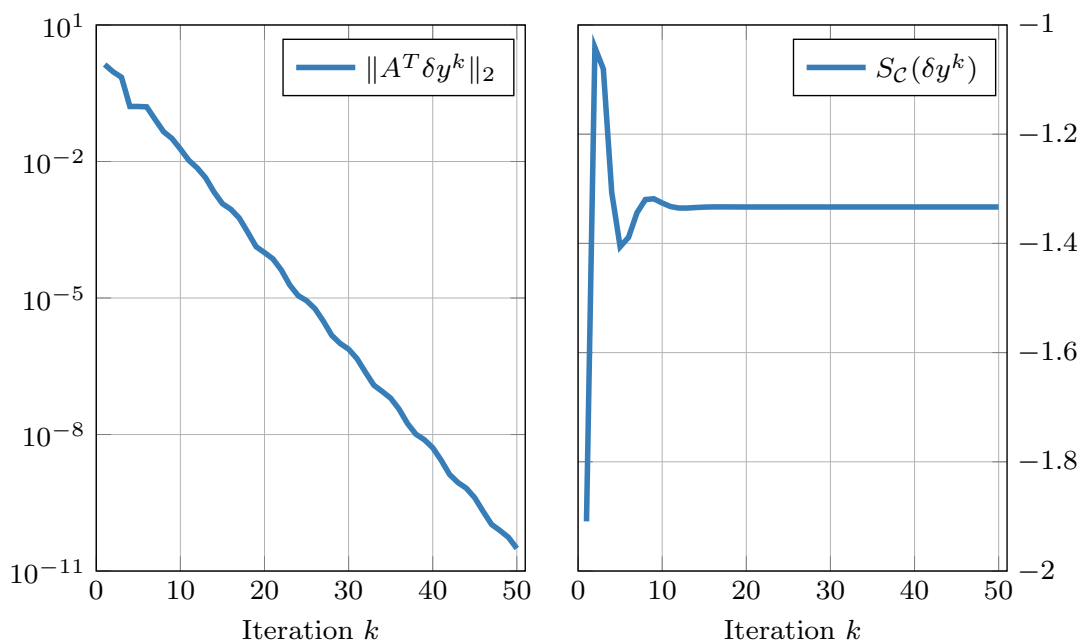

Fig. 2 Convergence of $\left\{\delta y^{k}\right\}_{k \in \mathbb{N}}$ to a certificate of primal infeasibility for problem (32) with $a=1, u_{1}=0$ and $u_{3}=3$.

Note that in this case the constraint set is $\mathcal{C}=\{0\} \times[1,3] \times[1,3]$. The sequence $\left\{\delta y^{k}\right\}_{k \in \mathbb{N}}$ generated by Algorithm 1 converges to $\delta y=(2 / 3,-2 / 3,-2 / 3)$, and we have

$$
A^{T} \delta y=0 \quad \text { and } \quad S_{\mathcal{C}}(\delta y)=-4 / 3<0 .
$$

According to Proposition 3.1(i), $\delta y$ is a certificate of primal infeasibility for the problem. Figure 2 shows convergence of $\left\{\delta y^{k}\right\}_{k \in \mathbb{N}}$ to a certificate of primal infeasibility.

\section{Dual infeasibility.}

We set the parameters to

$$
a=0, \quad u_{1}=2, \quad u_{3}=+\infty .
$$

The constraint set has the form $\mathcal{C}=\mathcal{B} \times \mathcal{K}_{b}$ with

$$
\mathcal{B}=[0,2] \times[1,3], \quad \mathcal{K}=\mathbb{R}_{+}, \quad b=1,
$$

and the constraint matrix $A$ can be written as

$$
A=\left[\begin{array}{l}
A_{1} \\
A_{2}
\end{array}\right] \quad \text { with } A_{1}=\left[\begin{array}{ll}
1 & 0 \\
1 & 0
\end{array}\right] \text { and } A_{2}=\left[\begin{array}{ll}
0 & 1
\end{array}\right] .
$$

The sequence $\left\{\delta x^{k}\right\}_{k \in \mathbb{N}}$ generated by Algorithm 1 converges to $\delta x=\left(0, \frac{1}{2}\right)$, and we have

$$
P \delta x=0, \quad A_{1} \delta x=0, \quad A_{2} \delta x=\frac{1}{2} \in \mathcal{K}, \quad\langle q, \delta x\rangle=-\frac{1}{2}<0 .
$$

According to Proposition 3.1(ii), $\delta x$ is a certificate of dual infeasibility of the problem. Figure 3 shows convergence of $\left\{\delta x^{k}\right\}_{k \in \mathbb{N}}$ to a certificate of dual infeasibility, where $\operatorname{dist}_{\mathcal{C}^{\infty}}$ denotes the Euclidean distance to the set $\mathcal{C}^{\infty}=\{0\} \times\{0\} \times \mathbb{R}_{+}$. 

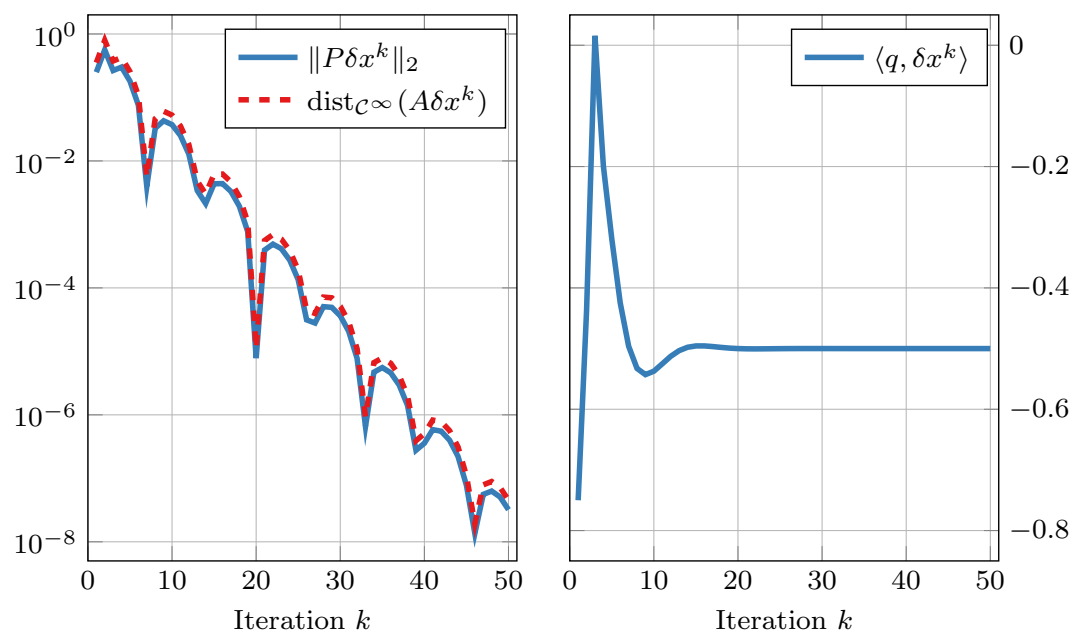

Fig. 3 Convergence of $\left\{\delta x^{k}\right\}_{k \in \mathbb{N}}$ to a certificate of dual infeasibility for problem (32) with $a=0, u_{1}=2$ and $u_{3}=+\infty$.

Simultaneous primal and dual infeasibility.

We set

$$
a=0, \quad u_{1}=0, \quad u_{3}=+\infty .
$$

The constraint set has the form $\mathcal{C}=\mathcal{B} \times \mathcal{K}_{b}$ with

$$
\mathcal{B}=\{0\} \times[1,3], \quad \mathcal{K}=\mathbb{R}_{+}, \quad b=1,
$$

and the constraint matrix $A$ can be written as in (33). The sequences $\left\{\delta x^{k}\right\}_{k \in \mathbb{N}}$ and $\left\{\delta y^{k}\right\}_{k \in \mathbb{N}}$ generated by Algorithm 1 converge to $\delta x=\left(0, \frac{1}{2}\right)$ and $\delta y=\left(\frac{1}{2},-\frac{1}{2}, 0\right)$, respectively. If we partition $\delta y$ as $\delta y=\left(\delta y_{1}, \delta y_{2}\right)$ with $\delta y_{1}=\left(\frac{1}{2},-\frac{1}{2}\right)$ and $\delta y_{2}=0$, then we have

$$
A^{T} \delta y=0, \quad \delta y_{2}=0 \in \mathcal{K}^{\circ}, \quad S_{\mathcal{B}}\left(\delta y_{1}\right)+\left\langle b, \delta y_{2}\right\rangle=-\frac{1}{2}<0,
$$

and

$$
P \delta x=0, \quad A_{1} \delta x=0, \quad A_{2} \delta x=\frac{1}{2} \in \mathcal{K}, \quad\langle q, \delta x\rangle=-\frac{1}{2}<0 .
$$

Therefore, $\delta x$ and $\delta y$ are certificates that the problem is simultaneously primal and dual infeasible. Figure 4 shows convergence of $\left\{\delta y^{k}\right\}_{k \in \mathbb{N}}$ and $\left\{\delta x^{k}\right\}_{k \in \mathbb{N}}$ to certificates of primal and dual infeasibility, respectively.

\subsection{Infeasible SDPs from SDPLIB}

We next demonstrate the asymptotic behavior of Algorithm 1 on two infeasible SDPs from the benchmark library SDPLIB [38]. The problems are given in the following form

$$
\min _{(x, z)} q^{T} x \quad \text { s.t. } \quad A x=z \quad \text { and } \quad z \in \mathcal{S}_{b}^{m},
$$



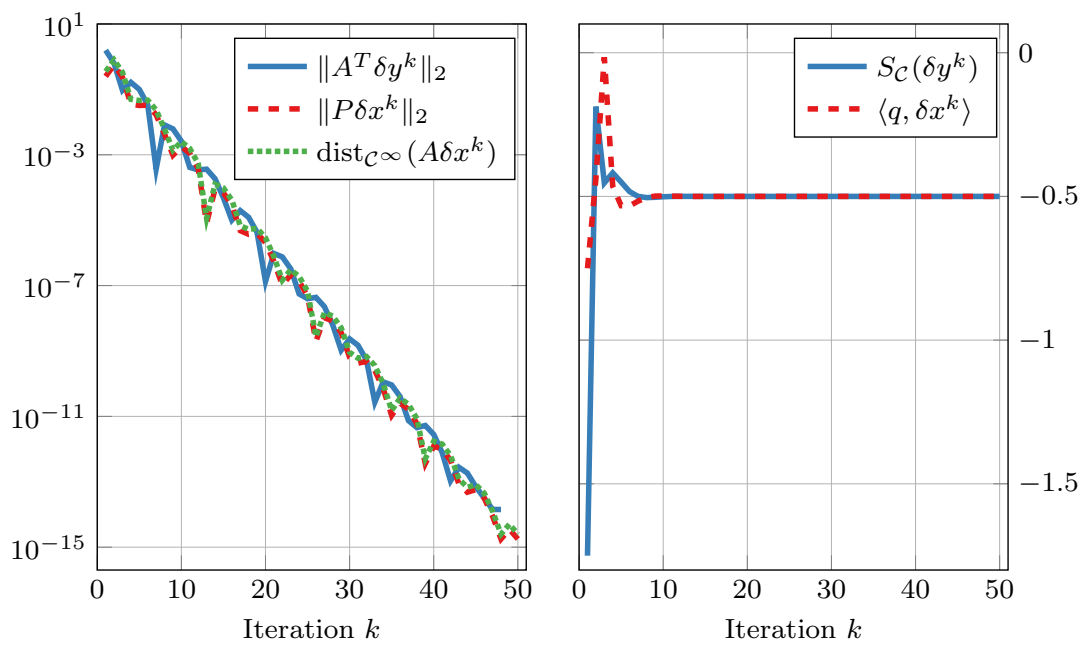

Fig. 4 Convergence of $\left\{\delta y^{k}\right\}_{k \in \mathbb{N}}$ and $\left\{\delta x^{k}\right\}_{k \in \mathbb{N}}$ to certificates of primal and dual infeasibility, respectively, for problem (32) with $a=0, u_{1}=0$ and $u_{3}=+\infty$.

where $\mathcal{S}^{m}$ denotes the vectorized form of $\mathbb{S}_{+}^{m}$, i.e., $z \in \mathcal{S}^{m}$ is equivalent to $\operatorname{mat}(z) \in \mathbb{S}_{+}^{m}$, and $\mathcal{S}_{b}^{m}:=\mathcal{S}^{m}+\{b\}$.

Let $X \in \mathbb{S}^{m}$ have the following eigenvalue decomposition

$$
X=U \operatorname{diag}\left(\lambda_{1}, \ldots, \lambda_{m}\right) U^{T}
$$

Then the projection of $X$ onto $\mathbb{S}_{+}^{m}$ is

$$
\Pi_{\mathbb{S}_{+}^{m}}(X)=U \operatorname{diag}\left(\max \left(\lambda_{1}, 0\right), \ldots, \max \left(\lambda_{m}, 0\right)\right) U^{T}
$$

Primal infeasible SDPs.

The primal infeasible problem infp1 from SDPLIB has decision variables $x \in \mathbb{R}^{10}$ and $z \in \mathcal{S}^{30}$. We run Algorithm 1 with parameters $\alpha=1$ and $\rho=\sigma=0.1$ from the initial iterate $\left(x^{0}, z^{0}, y^{0}\right)=(0,0,0)$. Figure 5 shows convergence of $\left\{\delta y^{k}\right\}_{k \in \mathbb{N}}$ to a certificate of primal infeasibility, where $\operatorname{dist}_{\mathcal{S}^{m}}(y)$ denotes the spectral norm distance of mat $(y)$ to the positive semidefinite cone $\mathbb{S}_{+}^{m}$.

Dual infeasible SDPs.

Dual infeasible problem infd1 from SDPLIB has decision variables $x \in \mathbb{R}^{10}$ and $z \in \mathcal{S}^{30}$. We run Algorithm 1 with parameters $\alpha=1$ and $\rho=\sigma=0.001$ from the initial iterate $\left(x^{0}, z^{0}, y^{0}\right)=(0,0,0)$. Figure 6 shows convergence of $\left\{\delta x^{k}\right\}_{k \in \mathbb{N}}$ to a certificate of dual infeasibility. 

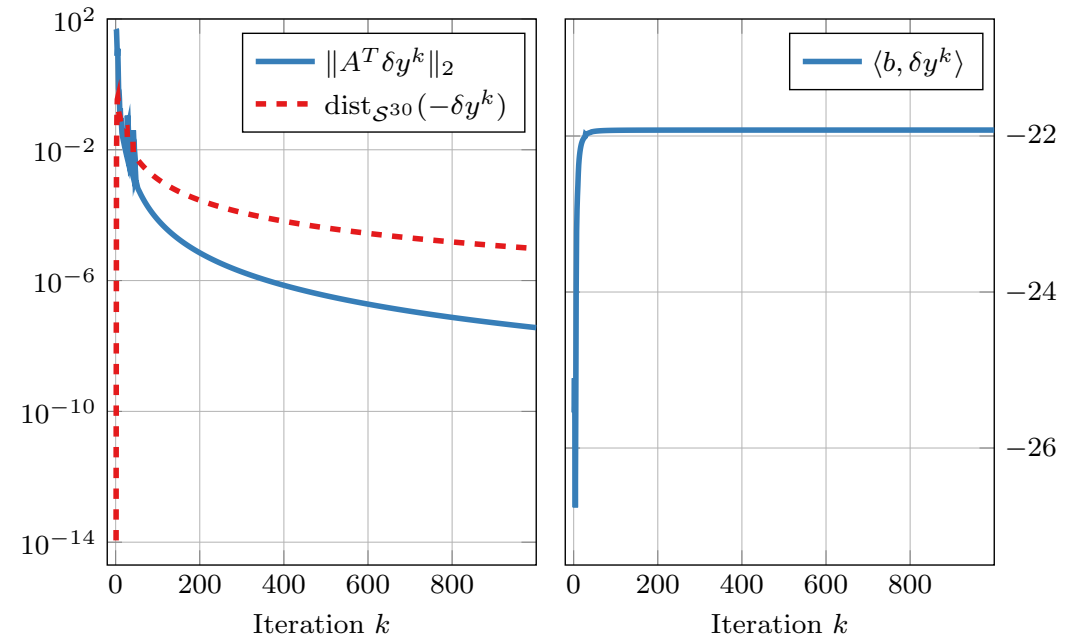

Fig. 5 Convergence of $\left\{\delta y^{k}\right\}_{k \in \mathbb{N}}$ to a certificate of primal infeasibility for problem infp1 from SDPLIB.

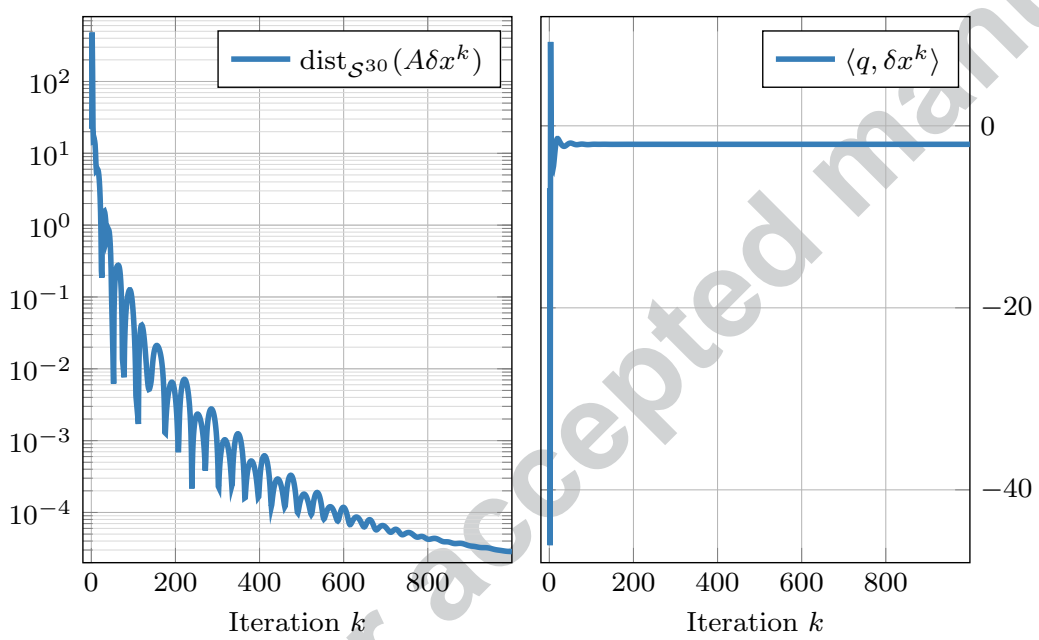

Fig. 6 Convergence of $\left\{\delta x^{k}\right\}_{k \in \mathbb{N}}$ to a certificate of dual infeasibility for problem infd1 from SDPLIB.

\subsection{Infeasible SDPs with no Certificate}

Consider the following feasibility problem [39, Ex. 5]

$$
\min _{\left(x_{1}, x_{2}\right)} 0 \text { s.t. }\left[\begin{array}{ccc}
x_{1} & 1 & 0 \\
1 & x_{2} & 0 \\
0 & 0 & -x_{1}
\end{array}\right] \succeq 0,
$$


noting that it is primal infeasible by inspection. If we write the constraint set in $(34)$ as

$$
\underbrace{\left[\begin{array}{ccc}
1 & 0 & 0 \\
0 & 0 & 0 \\
0 & 0 & -1
\end{array}\right]}_{A_{1}} x_{1}+\underbrace{\left[\begin{array}{ccc}
0 & 0 & 0 \\
0 & 1 & 0 \\
0 & 0 & 0
\end{array}\right]}_{A_{2}} x_{2}+\underbrace{\left[\begin{array}{lll}
0 & 1 & 0 \\
1 & 0 & 0 \\
0 & 0 & 0
\end{array}\right]}_{A_{0}} \succeq 0
$$

and denote by $A=\left[\operatorname{vec}\left(A_{1}\right) \operatorname{vec}\left(A_{2}\right)\right]$ and $b=-\operatorname{vec}\left(A_{0}\right)$, then the constraint can be written as $A x \in \mathcal{S}_{b}^{3}$, where $\mathcal{S}^{3}$ denotes the vectorized form of $\mathbb{S}_{+}^{3}$. If we define $Y:=\operatorname{mat}(y)$, then the primal infeasibility condition (5) for the problem above amounts to

$$
Y_{11}-Y_{33}=0, \quad Y_{22}=0, \quad Y_{12}<0, \quad Y \preceq 0,
$$

where $Y_{i j}$ denotes the element of $Y \in \mathbb{S}^{3}$ in the $i$-th row and $j$-th column. Given that $Y \preceq 0$ and $Y_{22}=0$ imply $Y_{12}=0$, the system above is infeasible as well. Note that $Y=0$ is feasible for the dual of problem (34) and problem (34) is thus not dual infeasible.

We next show that $\left(\delta x^{k}, \delta Z^{k}, \delta Y^{k}\right) \rightarrow(0,0,0)$, where $\delta Z^{k}:=\operatorname{mat}\left(\delta z^{k}\right)$ and $\delta Y^{k}:=\operatorname{mat}\left(\delta y^{k}\right)$. Set

$$
x^{k}=\left(\left(1+\rho \sigma^{-1}\right) \varepsilon, \varepsilon^{-1}\right) \quad \text { and } \quad V^{k}:=\operatorname{mat}\left(v^{k}\right)=\operatorname{diag}\left(\varepsilon, \varepsilon^{-1}, 0\right),
$$

where $\varepsilon>0$. Iteration (12) then produces the following iterates

$$
Z^{k}=V^{k}, \quad \tilde{x}^{k}=\left(\varepsilon, \varepsilon^{-1}\right), \quad \tilde{Z}^{k}=\operatorname{diag}\left(\varepsilon, \varepsilon^{-1},-\varepsilon\right),
$$

and thus we have

$$
\begin{aligned}
\delta x^{k+1} & =\alpha\left(\tilde{x}^{k}-x^{k}\right)=\alpha\left(-\rho \sigma^{-1} \varepsilon, 0\right) \\
\delta V^{k+1} & =\alpha\left(\tilde{Z}^{k}-Z^{k}\right)=\alpha \operatorname{diag}(0,0,-\varepsilon) .
\end{aligned}
$$

By taking $\varepsilon$ arbitrarily small, we can make $\left(\delta x^{k+1}, \delta V^{k+1}\right)$ arbitrarily close to zero, which according to Lemma 5.1 means that $\left(\delta x^{k}, \delta V^{k}\right) \rightarrow(\delta x, \delta V)=(0,0)$, and according to Proposition 5.3 the optimality conditions (3) are satisfied in the limit. However, the sequence $\left\{x^{k}, Z^{k}, Y^{k}\right\}_{k \in \mathbb{N}}$ has no limit point; otherwise, such a point would be a certificate for optimality of the problem. Let $T$ denote the fixed-point operator mapping $\left(x^{k}, V^{k}\right)$ to $\left(x^{k+1}, V^{k+1}\right)$. Since $(\delta x, \delta V) \in \operatorname{cl} \operatorname{ran}(T-$ Id $)$ by definition, and $(\delta x, \delta V) \notin \operatorname{ran}(T-\mathrm{Id})$, this means that the set $\operatorname{ran}(T-\mathrm{Id})$ is not closed, and the distance from $(\delta x, \delta V)$ to $\operatorname{ran}(T-\mathrm{Id})$ is zero. In other words, the set

$$
\left\{\left[\begin{array}{ccc}
x_{1} & 1 & 0 \\
1 & x_{2} & 0 \\
0 & 0 & -x_{1}
\end{array}\right]:\left(x_{1}, x_{2}\right) \in \mathbb{R}^{2}\right\}
$$

and the semidefinite cone $\mathbb{S}_{+}^{3}$ do not intersect, but are not strongly separable.

We run Algorithm 1 with parameters $\alpha=\rho=\sigma=1$ from the initial iterate $\left(x^{0}, Z^{0}, Y^{0}\right)=(0,0,0)$. Figure 7 shows convergence of residuals $\left\|A x^{k}-z^{k}\right\|_{2}$ and $\left\|A^{T} y^{k}\right\|_{2}$ to zero. 


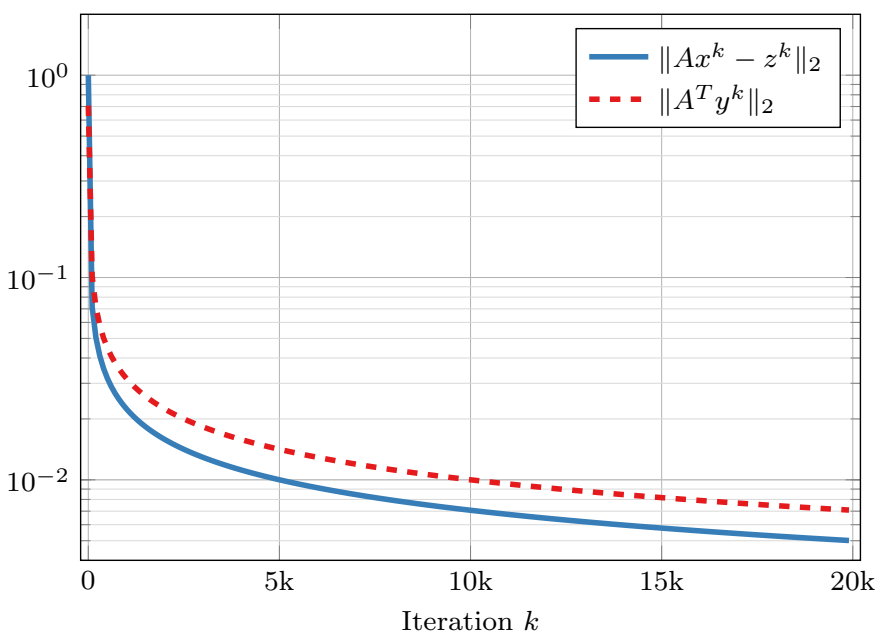

Fig. 7 Convergence of residuals $\left\|A x^{k}-z^{k}\right\|_{2}$ and $\left\|A^{T} y^{k}\right\|_{2}$ for problem (34).

Remark 6.1 Let $\varepsilon>0$. Consider the following perturbation to problem (34):

$$
\min _{\left(x_{1}, x_{2}\right)} \quad 0 \text { s.t. }\left[\begin{array}{ccc}
x_{1} & 1 & 0 \\
1 & x_{2} & 0 \\
0 & 0 & -x_{1}
\end{array}\right] \succeq-\varepsilon I .
$$

This problem is feasible since the constraint above is satisfied for $x_{1}=0$ and $x_{2}=1 / \varepsilon-\varepsilon$.

Consider now the following problem:

$$
\min _{\left(x_{1}, x_{2}\right)} \quad 0 \text { s.t. }\left[\begin{array}{ccc}
x_{1} & 1 & 0 \\
1 & x_{2} & 0 \\
0 & 0 & -x_{1}
\end{array}\right] \succeq \varepsilon I .
$$

This problem is strongly infeasible since the vector $\bar{y}=\operatorname{vec}(\operatorname{diag}(-1,0,-1))$ satisfies the primal infeasibility condition (5).

These two examples show that an infinitesimally small perturbation to problem (34) can make the problem feasible or strongly infeasible.

\section{Conclusions}

We have analyzed the asymptotic behavior of ADMM for a class of convex optimization problems, and have shown that if the problem is primal and/or dual strongly infeasible, then the sequence of successive differences of the algorithm's iterates converge to a certificate of infeasibility. Based on these results, we have proposed termination criteria for detecting primal and dual infeasibility, providing for the first time a set of reliable and generic stopping criteria for ADMM applicable to infeasible convex problems. We have also provided 
numerical examples to demonstrate different asymptotic behaviors of the algorithm's iterates.

Acknowledgements We are grateful to Walaa Moursi for helpful comments and pointing out additional references. This work was supported by the People Programme (Marie Curie Actions) of the European Union Seventh Framework Programme (FP7/2007-2013) under REA grant agreement no. 607957 (TEMPO).

\section{Appendix A Supporting Results}

Lemma A.1 The first-order optimality conditions for problem (2) are conditions (3).

Proof We first rewrite problem (2) in the form

$$
\min _{(x, z)}\left(\frac{1}{2} x^{T} P x+q^{T} x+\mathcal{I}_{\mathcal{C}}(z)\right) \quad \text { s.t. } \quad A x=z,
$$

and then form its Lagrangian,

$$
\mathcal{L}(x, z, y):=\frac{1}{2} x^{T} P x+q^{T} x+\mathcal{I}_{\mathcal{C}}(z)+y^{T}(A x-z) .
$$

Provided that the problem satisfies certain constraint qualification [15, Cor. 26.3], its solution can be characterized via a saddle point of (35). Therefore, the first-order optimality conditions can be written as [29, Ex. 11.52]

$$
\begin{aligned}
z & \in \mathcal{C} \\
0 & =-\nabla_{x} \mathcal{L}(x, z, y)=-\left(P x+q+A^{T} y\right) \\
N_{\mathcal{C}}(z) & \ni-\nabla_{z} \mathcal{L}(x, z, y)=y \\
0 & =\nabla_{y} \mathcal{L}(x, z, y)=A x-z .
\end{aligned}
$$

Lemma A.2 The dual of problem (1) is given by problem (4).

Proof The dual function can be derived from the Lagrangian (35) as follows:

$$
\begin{aligned}
g(y) & :=\inf _{(x, z)} \mathcal{L}(x, z, y) \\
& =\inf _{x}\left\{\frac{1}{2} x^{T} P x+\left(A^{T} y+q\right)^{T} x\right\}+\inf _{z \in \mathcal{C}}\left\{-y^{T} z\right\} \\
& =\inf _{x}\left\{\frac{1}{2} x^{T} P x+\left(A^{T} y+q\right)^{T} x\right\}-\sup _{z \in \mathcal{C}}\left\{y^{T} z\right\} .
\end{aligned}
$$

Note that the minimum of the Lagrangian over $x$ is attained when $P x+A^{T} y+q=0$, and the second term in the last line is $S_{\mathcal{C}}(y)$. The dual problem, defined as the problem of maximizing the dual function, can then be written in the form (4), where the conic constraint on $y$ is just the restriction of $y$ to the domain of $S_{\mathcal{C}}$ [31, p.112 and Cor. 14.2.1].

Lemma A.3 For any vectors $v \in \mathbb{R}^{n}, b \in \mathbb{R}^{n}$ and a nonempty, closed, and convex cone $\mathcal{K} \subseteq \mathbb{R}^{n}$
(i) $\Pi_{\mathcal{K}_{b}}(v)=b+\Pi_{\mathcal{K}}(v-b)$
(ii) $\left(\operatorname{Id}-\Pi_{\mathcal{K}_{b}}\right)(v)=\Pi_{\mathcal{K}^{\circ}}(v-b)$.
(iii) $\left\langle\Pi_{\mathcal{K}_{b}}(v),\left(\operatorname{Id}-\Pi_{\mathcal{K}_{b}}\right)(v)\right\rangle=\left\langle b, \Pi_{\mathcal{K}^{\circ}}(v-b)\right\rangle$
(iv) $\left\langle\Pi_{\mathcal{K}}(v), v\right\rangle=\left\|\Pi_{\mathcal{K}}(v)\right\|^{2}$
(v) $S_{\mathcal{K}_{b}}\left(\Pi_{\mathcal{K}^{\circ}}(v)\right)=\left\langle b, \Pi_{\mathcal{K}^{\circ}}(v)\right\rangle$. 
Proof Part (i) is from [15, Prop. 28.1(i)].

(ii): From part (i) we have

$$
\left(\operatorname{Id}-\Pi_{\mathcal{K}_{b}}\right)(v)=v-b-\Pi_{\mathcal{K}}(v-b)=\Pi_{\mathcal{K}^{\circ}}(v-b),
$$

where the second equality follows from the Moreau decomposition [15, Thm. 6.29].

(iii): Follows directly from parts (i) and (ii), and the Moreau decomposition.

(iv): From the Moreau decomposition, we have

$$
\left\langle\Pi_{\mathcal{K}}(v), v\right\rangle=\left\langle\Pi_{\mathcal{K}}(v), \Pi_{\mathcal{K}}(v)+\Pi_{\mathcal{K}^{\circ}}(v)\right\rangle=\left\|\Pi_{\mathcal{K}}(v)\right\|^{2} .
$$

(v): Since the support function of $\mathcal{K}$ evaluated at any point in $\mathcal{K}^{\circ}$ is zero, we have

$$
S_{\mathcal{K}_{b}}\left(\Pi_{\mathcal{K}^{\circ}}(v)\right)=\left\langle b, \Pi_{\mathcal{K}^{\circ}}(v)\right\rangle+S_{\mathcal{K}}\left(\Pi_{\mathcal{K}^{\circ}}(v)\right)=\left\langle b, \Pi_{\mathcal{K}^{\circ}}(v)\right\rangle .
$$

Lemma A.4 Suppose that $\mathcal{K} \subseteq \mathbb{R}^{n}$ is a nonempty, closed, and convex cone and for some sequence $\left\{v^{k}\right\}_{k \in \mathbb{N}}$, where $v^{k} \in \mathbb{R}^{n}$, we denote by $\delta v:=\lim _{k \rightarrow \infty} \frac{1}{k} v^{k}$, assuming that the limit exists. Then for any $b \in \mathbb{R}^{n}$,

$$
\lim _{k \rightarrow \infty} \frac{1}{k} \Pi_{\mathcal{K}_{b}}\left(v^{k}\right)=\lim _{k \rightarrow \infty} \frac{1}{k} \Pi_{\mathcal{K}}\left(v^{k}-b\right)=\Pi_{\mathcal{K}}(\delta v) .
$$

Proof Write the limit as

$$
\begin{aligned}
\lim _{k \rightarrow \infty} \frac{1}{k} \Pi_{\mathcal{K}_{b}}\left(v^{k}\right) & =\lim _{k \rightarrow \infty} \frac{1}{k}\left(b+\Pi_{\mathcal{K}}\left(v^{k}-b\right)\right) \\
& =\lim _{k \rightarrow \infty} \Pi_{\mathcal{K}}\left(\frac{1}{k}\left(v^{k}-b\right)\right) \\
& =\Pi_{\mathcal{K}}\left(\lim _{k \rightarrow \infty} \frac{1}{k} v^{k}\right),
\end{aligned}
$$

where the first equality uses Lemma A.3(i), and the second and third follow from the positive homogeneity [15, Prop. 28.22] and continuity [15, Prop. 4.8] of $\Pi_{\mathcal{K}}$, respectively.

Lemma A.5 Suppose that $\mathcal{B} \subseteq \mathbb{R}^{n}$ is a nonempty, convex and compact set and for some sequence $\left\{v^{k}\right\}_{k \in \mathbb{N}}$, where $v^{k} \in \mathbb{R}^{n}$, we denote by $\delta v:=\lim _{k \rightarrow \infty} \frac{1}{k} v^{k}$, assuming that the limit exists. Then

$$
\lim _{k \rightarrow \infty} \frac{1}{k}\left\langle v^{k}, \Pi_{\mathcal{B}}\left(v^{k}\right)\right\rangle=\lim _{k \rightarrow \infty}\left\langle\delta v, \Pi_{\mathcal{B}}\left(v^{k}\right)\right\rangle=S_{\mathcal{B}}(\delta v) .
$$

Proof Let $z^{k}:=\Pi_{\mathcal{B}}\left(v^{k}\right)$. We have the following inclusion [15, Prop. 6.46]

$$
v^{k}-z^{k} \in N_{\mathcal{B}}\left(z^{k}\right) \text {, }
$$

which, due to $\left[15\right.$, Thm. 16.23], and the facts that $S_{\mathcal{B}}$ is the Fenchel conjugate of $\mathcal{I}_{\mathcal{B}}$ and $N_{\mathcal{B}}$ is the subdifferential of $\mathcal{I}_{\mathcal{B}}$, is equivalent to

$$
\left\langle\frac{1}{k}\left(v^{k}-z^{k}\right), z^{k}\right\rangle=S_{\mathcal{B}}\left(\frac{1}{k}\left(v^{k}-z^{k}\right)\right) .
$$

Taking the limit of the identity above, we obtain

$$
\lim _{k \rightarrow \infty}\left\langle\frac{1}{k}\left(v^{k}-z^{k}\right), z^{k}\right\rangle=\lim _{k \rightarrow \infty} S_{\mathcal{B}}\left(\frac{1}{k}\left(v^{k}-z^{k}\right)\right)=S_{\mathcal{B}}\left(\lim _{k \rightarrow \infty} \frac{1}{k}\left(v^{k}-z^{k}\right)\right)=S_{\mathcal{B}}(\delta v),
$$

where the second equality follows from the continuity of $S_{\mathcal{B}}$ [15, Ex. 11.2], and the third from the compactness of $\mathcal{B}$. Since $\left\{z^{k}\right\}_{k \in \mathbb{N}}$ remains in the compact set $\mathcal{B}$, we can derive the following relation from (36):

$$
\begin{aligned}
\left|S_{\mathcal{B}}(\delta v)-\lim _{k \rightarrow \infty}\left\langle\delta v, z^{k}\right\rangle\right| & =\left|\lim _{k \rightarrow \infty}\left\langle\frac{1}{k}\left(v^{k}-z^{k}\right), z^{k}\right\rangle-\left\langle\delta v, z^{k}\right\rangle\right| \\
& =\left|\lim _{k \rightarrow \infty}\left\langle\frac{1}{k} v^{k}-\delta v, z^{k}\right\rangle-\frac{1}{k}\left\langle z^{k}, z^{k}\right\rangle\right| \\
& \leq \lim _{k \rightarrow \infty} \underbrace{\left\|\frac{1}{k} v^{k}-\delta v\right\|}_{\rightarrow 0}\left\|z^{k}\right\|+\frac{1}{k}\left\|z^{k}\right\|^{2} \\
& =0
\end{aligned}
$$


where the third row follows from the triangle and Cauchy-Schwarz inequalities, and the fourth from the compactness of $\mathcal{B}$. Finally, we can derive the following identity from (36):

$$
S_{\mathcal{B}}(\delta v)=\lim _{k \rightarrow \infty}\left\langle\frac{1}{k}\left(v^{k}-z^{k}\right), z^{k}\right\rangle=\lim _{k \rightarrow \infty}\left\langle\frac{1}{k} v^{k}, z^{k}\right\rangle-\underbrace{\frac{1}{k}\left\|z^{k}\right\|^{2}}_{\rightarrow 0} .
$$

This concludes the proof.

\section{References}

1. Parikh, N., Boyd, S.: Proximal algorithms. Foundations and Trends in Optimization 1(3), 123-231 (2013). DOI 10.1561/2400000003

2. Bauschke, H.H., Borwein, J.M.: On projection algorithms for solving convex feasibility problems. SIAM Review 38(3), 367-426 (1996). DOI 10.1137/S0036144593251710

3. Bauschke, H.H., Combettes, P.L., Luke, D.R.: Finding best approximation pairs relative to two closed convex sets in Hilbert spaces. Journal of Approximation Theory 127(2), 178-192 (2004). DOI 10.1016/j.jat.2004.02.006

4. Boley, D.: Local linear convergence of the alternating direction method of multipliers on quadratic or linear programs. SIAM Journal on Optimization 23(4), 2183-2207 (2013). DOI $10.1137 / 120878951$

5. O'Donoghue, B., Chu, E., Parikh, N., Boyd, S.: Conic optimization via operator splitting and homogeneous self-dual embedding. Journal of Optimization Theory and Applications 169(3), 1042-1068 (2016). DOI 10.1007/s10957-016-0892-3

6. Zheng, Y., Fantuzzi, G., Papachristodoulou, A., Goulart, P., Wynn, A.: Chordal decomposition in operator-splitting methods for sparse semidefinite programs. Mathematical Programming (2019). DOI 10.1007/s10107-019-01366-3

7. O'Donoghue, B., Stathopoulos, G., Boyd, S.: A splitting method for optimal control IEEE Transactions on Control Systems Technology 21(6), 2432-2442 (2013). DOI 10.1109/TCST.2012.2231960

8. Jerez, J., Goulart, P., Richter, S., Constantinides, G., Kerrigan, E., Morari, M.: Embedded online optimization for model predictive control at megahertz rates. IEEE Transactions on Automatic Control 59(12), 3238-3251 (2014). DOI 10.1109/TAC.2014.2351991

9. Banjac, G., Stellato, B., Moehle, N., Goulart, P., Bemporad, A., Boyd, S.: Embedded code generation using the OSQP solver. In: IEEE Conference on Decision and Control (CDC), pp. 1906-1911 (2017). DOI 10.1109/CDC.2017.8263928

10. Beck, A., Teboulle, M.: Fast gradient-based algorithms for constrained total variation image denoising and deblurring problems. IEEE Transactions on Image Processing 18(11), 2419-2434 (2009). DOI 10.1109/TIP.2009.2028250

11. Combettes, P.L., Wajs, V.R.: Signal recovery by proximal forward-backward splitting. Multiscale Modeling \& Simulation 4(4), 1168-1200 (2005). DOI 10.1137/050626090

12. Combettes, P.L., Pesquet, J.C.: Proximal splitting methods in signal processing. Springer Optimization and Its Applications 49, 185-212 (2011). DOI 10.1007/ 978-1-4419-9569-8_10

13. Boyd, S., Parikh, N., Chu, E., Peleato, B., Eckstein, J.: Distributed optimization and statistical learning via the alternating direction method of multipliers. Foundations and Trends in Machine Learning 3(1), 1-122 (2011). DOI 10.1561/2200000016

14. Stathopoulos, G., Shukla, H., Szucs, A., Pu, Y., Jones, C.: Operator splitting methods in control. Foundations and Trends in Systems and Control 3(3), 249-362 (2016). DOI $10.1561 / 2600000008$

15. Bauschke, H.H., Combettes, P.L.: Convex Analysis and Monotone Operator Theory in Hilbert Spaces. Springer Science \& Business Media (2011). DOI 10.1007/ 978-1-4419-9467-7

16. Ghadimi, E., Teixeira, A., Shames, I., Johansson, M.: Optimal parameter selection for the alternating direction method of multipliers (ADMM): quadratic problems. IEEE Transactions on Automatic Control 60(3), 644-658 (2015). DOI 10.1109/TAC.2014. 2354892 
17. Giselsson, P., Boyd, S.: Linear convergence and metric selection for Douglas-Rachford splitting and ADMM. IEEE Transactions on Automatic Control 62(2), 532-544 (2017). DOI 10.1109/TAC.2016.2564160

18. Banjac, G., Goulart, P.: Tight global linear convergence rate bounds for operator splitting methods. IEEE Transactions on Automatic Control 63(12), 4126-4139 (2018). DOI 10.1109/TAC.2018.2808442

19. Naik, V.V., Bemporad, A.: Embedded mixed-integer quadratic optimization using accelerated dual gradient projection. In: IFAC World Congress, pp. 10,723-10,728 (2017). DOI 10.1016/j.ifacol.2017.08.2235

20. Eckstein, J., Bertsekas, D.P.: On the Douglas-Rachford splitting method and the proximal point algorithm for maximal monotone operators. Mathematical Programming 55(1), 293-318 (1992). DOI 10.1007/BF01581204

21. Bauschke, H.H., Dao, M.N., Moursi, W.M.: The Douglas-Rachford algorithm in the affine-convex case. Operations Research Letters 44(3), 379-382 (2016). DOI 10.1016/ j.orl.2016.03.010

22. Bauschke, H.H., Moursi, W.M.: The Douglas-Rachford algorithm for two (not necessarily intersecting) affine subspaces. SIAM Journal on Optimization 26(2), 968-985 (2016). DOI 10.1137/15M1016989

23. Bauschke, H.H., Moursi, W.M.: On the Douglas-Rachford algorithm. Mathematical Programming 164(1), 263-284 (2017). DOI 10.1007/s10107-016-1086-3

24. Moursi, W.M.: The Douglas-Rachford operator in the possibly inconsistent case: static properties and dynamic behaviour. Ph.D. thesis, University of British Columbia (2016)

25. Raghunathan, A.U., Di Cairano, S.: Infeasibility detection in alternating direction method of multipliers for convex quadratic programs. In: IEEE Conference on Decision and Control (CDC), pp. 5819-5824 (2014). DOI 10.1109/CDC.2014.7040300

26. Toh, K.C.: An inexact primal-dual path following algorithm for convex quadratic SDP. Mathematical Programming 112(1), 221-254 (2008). DOI 10.1007/s10107-006-0088-y

27. Henrion, D., Malick, J.: Projection Methods in Conic Optimization, pp. 565-600. Springer US (2012). DOI 10.1007/978-1-4614-0769-0_20

28. Stellato, B., Banjac, G., Goulart, P., Bemporad, A., Boyd, S.: OSQP: an operator splitting solver for quadratic programs. arXiv:1711.08013 (2018)

29. Rockafellar, R.T., Wets, R.J.B.: Variational Analysis. Grundlehren der mathematischen Wissenschaften. Springer (1998). DOI 10.1007/978-3-642-02431-3

30. Lourenço, B.F., Muramatsu, M., Tsuchiya, T.: Weak infeasibility in second order cone programming. Optimization Letters 10(8), 1743-1755 (2016). DOI 10.1007/ s11590-015-0982-4

31. Rockafellar, R.T.: Convex Analysis. Princeton University Press, USA (1970)

32. Boyd, S., Vandenberghe, L.: Convex Optimization. Cambridge University Press (2004). DOI 10.1017/CBO9780511804441

33. Gabay, D.: Applications of the method of multipliers to variational inequalities. Studies in Mathematics and its Applications 15(C), 299-331 (1983). DOI 10.1016/ S0168-2024(08)70034-1

34. Giselsson, P., Fält, M., Boyd, S.: Line search for averaged operator iteration. In: IEEE Conference on Decision and Control (CDC), pp. 1015-1022 (2016). DOI 10.1109/CDC. 2016.7798401

35. Lions, P., Mercier, B.: Splitting algorithms for the sum of two nonlinear operators. SIAM Journal on Numerical Analysis 16(6), 964-979 (1979). DOI 10.1137/0716071

36. Pazy, A.: Asymptotic behavior of contractions in Hilbert space. Israel Journal of Mathematics 9(2), 235-240 (1971). DOI 10.1007/BF02771588

37. Baillon, J.B., Bruck, R.E., Reich, S.: On the asymptotic behavior of nonexpansive mappings and semigroups in Banach spaces. Houston Journal of Mathematics 4(1), 1-9 (1978)

38. Borchers, B.: SDPLIB 1.2, a library of semidefinite programming test problems. Optimization Methods and Software 11(1), 683-690 (1999). DOI 10.1080/ 10556789908805769

39. Ramana, M.V.: An exact duality theory for semidefinite programming and its complexity implications. Mathematical Programming 77(1), 129-162 (1997). DOI $10.1007 / \mathrm{BF} 02614433$ 